\title{
ANLN-induced EZH2 upregulation promotes pancreatic cancer progression by mediating miR-218-5p/LASP1 signaling axis
}

Anbin Wang ${ }^{\dagger}$, Haisu Dai ${ }^{\dagger}$, Yi Gong, Chengcheng Zhang, Junjie Shu, Yuandeng Luo, Yan Jiang, Wei Liu and Ping Bie

\begin{abstract}
Background: Pancreatic cancer is a highly lethal malignancy with poor prognosis. Anillin (ANLN), an actin binding protein, is upregulated and plays an important role in many malignant tumors. However, the precise role of ANLN in pancreatic cancer remains unclear.

Methods: The expression of ANLN and its association with pancreatic cancer patient survival were analyzed using an online database and confirmed by immunohistochemistry. The ANLN protein expression in pancreatic cancer cell lines was detected by Western blot. Cell proliferation, colony formation and transwell assays in vitro and in vivo tumor growth were used to determine the role of ANLN in pancreatic cancer. Gene expression microarray analysis and a series of in vitro assays were used to elucidate the mechanisms of ANLN regulating pancreatic cancer progression.

Results: We found that the ANLN expression was significantly upregulated in pancreatic cancer tissues and cell lines. The high expression of ANLN was associated with tumor size, tumor differentiation, TNM stage, lymph node metastasis, distant metastasis and poor prognosis in pancreatic cancer. ANLN downregulation significantly inhibited cell proliferation, colony formation, migration, invasion and tumorigenicity in nude mice. Meanwhile, we found that ANLN knockdown inhibited several cell-cell adhesion related genes, including the gene encoding LIM and SH3 protein 1 (LASP1). LASP1 upregulation partially reversed the tumor-suppressive effect of ANLN downregulation on pancreatic cancer cell progression. Moreover, we found that ANLN downregulation induced the expression of miR218-5p which inhibited LASP1 expression through binding to its $3^{\prime} U T R$. We also found that ANLN-induced enhancer of zeste homolog $2(\mathrm{EZH} 2)$ upregulation was involved in regulating miR-218-5p/LASP1 signaling axis. EZH2 upregulation or miR-218-5p downregulation partially reversed the tumor-suppressive effect of ANLN downregulation on pancreatic cancer cell progression.
\end{abstract}

Conclusion: ANLN contributed to pancreatic cancer progression by regulating EZH2/miR-218-5p/LASP1 signaling axis. These findings suggest that ANLN may be a candidate therapeutic target in pancreatic cancer.

Keywords: Pancreatic cancer, ANLN, EZH2, miR-218-5p, LASP1

\footnotetext{
* Correspondence: liuwei@tmmu.edu.cn; bieping@medmail.com.cn

${ }^{+}$Anbin Wang and Haisu Dai contributed equally to this work.

Department of Hepatobiliary Surgery, Southwest Hospital, Third Military

Medical University (Army Medical University), Chongqing, China
}

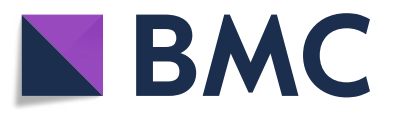

(c) The Author(s). 2019 Open Access This article is distributed under the terms of the Creative Commons Attribution 4.0 International License (http://creativecommons.org/licenses/by/4.0/), which permits unrestricted use, distribution, and reproduction in any medium, provided you give appropriate credit to the original author(s) and the source, provide a link to the Creative Commons license, and indicate if changes were made. The Creative Commons Public Domain Dedication waiver (http://creativecommons.org/publicdomain/zero/1.0/) applies to the data made available in this article, unless otherwise stated. 


\section{Background}

Pancreatic cancer is a fatal malignancy with a poor prognosis worldwide [1]. Due to occult onset and non-specific symptoms, $80 \%$ of patients diagnosed with pancreatic cancer are in advanced stages and have a 5-year survival rate of less than 5\% [2,3]. Despite ongoing advances for the survival rates noted in many cancers, such as colon cancer and breast cancer, the annual mortality rates for patients with pancreatic cancer remain almost equal to the incidence rates $[3,4]$. Thus, to improve the health outcomes of pancreatic cancer patients, more intensive efforts should be made to understand the molecular mechanisms underlying pancreatic cancer progression.

Anillin (ANLN), an actin binding protein, first identified in Drosophila, is located on chromosome 7p14.2 and encodes an actin-binding protein that consists of 1125 amino acids and plays an important role in cytokinesis [5-7]. In normal tissues, ANLN expression is higher in the placenta, brain and testis, and lower in the lung, heart, liver and spleen [8]. Many recent studies suggests that ANLN is upregulated in numerous cancer types, including cervical cancer, prostate cancer, anaplastic thyroid carcinoma, breast cancer, lung carcinogenesis, bladder urothelial carcinoma, pancreatic cancer and nasopharyngeal carcinoma [9-16]. Functionally, increasing evidence has indicated that ANLN is critical for growth and metastasis of cancer cells. For example, ANLN knockdown inhibited cell proliferation and metastasis and induced G2/M arrest in bladder urothelial carcinoma [16]. In non-small cell lung cancer cells, ANLN downregulation induced cell growth repression, and ANLN overexpression promoted cell motility [17]. Moreover, ANLN was upregulated in pancreatic cancer and was involved in miR-217-mediated cell proliferation and invasion [18]. Nevertheless, the mechanisms that underlie the role of ANLN in the regulation of pancreatic cancer progression have not been fully addressed.

LIM and SH3 protein 1 (LASP1), a structural scaffolding protein and adhesion adaptor protein, was initially identified in breast cancer and was located at 17q12 [19]. Previous studies showed that LASP1 was upregulated in many malignant tumors including nasopharyngeal carcinoma, breast cancer, glioblastoma and colorectal cancer and contributed to tumor proliferation, invasion and metastasis [20-23]. In addition, LASP1 is upregulated in pancreatic ductal adenocarcinoma and is essential for HIF $1 \alpha$-induced invasion and metastasis [24]. Moreover, our gene microarray analysis showed that ANLN downregulation repressed LASP1 expression. Whether LASP1 is involved in ANLN-induced pancreatic cancer progression is what we deal with in this study.

Recently, miRNAs have been shown to be deregulated in pancreatic cancer, affecting several steps of initiation and aggressiveness of the disease by directly regulating target genes expression [25]. Among these miRNAs, miR-218-5p was found to play pivotal roles in many malignant tumors [26-28]. For example, miR-218-5p upregulation repressed gastric cancer growth and metastasis by directly regulating CDK6/CyclinD1 [28]. Additionally, miR218 was downregulated in pancreatic cancer tissues when compared with adjacent normal tissues, and reduced miR218 was associated with poor prognosis of pancreatic cancer patients [29]. MiR-218 upregulation inhibited the proliferation and invasion and induced apoptosis of pancreatic cancer cells [30]. Interestingly, miR-218 could suppress cell proliferation, migration and invasion in gastric cancer and prostate cancer [31,32]. Moreover, our gene microarray analysis showed that ANLN downregulation induced miR-218 expression. Thus, it is necessary to study the roles of miR-218-5p in ANLN-induced pancreatic cancer progression.

Enhancer of zeste homolog 2 (EZH2), a histone methyltransferase, is highly amplified in human cancers and plays an important role in the development and progression of cancers [33, 34]. In 2011, Cao Q et al. first found that EZH2 contributed to miRNAs dysregulation via the PRC2/PRC1 axis [35]. In pancreatic cancer, EZH2 downregulation induced the expression of miR-139-5p via H3K27me3, thereby repressing the progression of pancreatic cancer [36]. In addition, EZH2-mediated formation of heterochromatin silenced miR-218 in human pancreatic ductal adenocarcinoma cells [37]. Our gene microarray analysis showed that ANLN downregulation inhibited EZH2 expression. However, the relationship between EZH2/miR-218 axis and ANLN in pancreatic cancer progression has not been studied before.

In this study, we showed that ANLN expression was upregulated in pancreatic cancer tissues and cell lines. A high expression level of ANLN was associated with poor prognosis of pancreatic cancer patients. ANLN downregulation inhibited pancreatic cancer cell proliferation, colony formation, migration and invasion. In addition, EZH2/ miR-218-5p/LASP1 signaling axis might be involved in ANLN-mediated cell proliferation, colony formation, migration and invasion in pancreatic cancer.

\section{Materials and methods}

\section{Cell lines and cell culture}

Five pancreatic cancer cell lines were acquired from the Cell Bank Type Culture Collection of the Chinese Academy of Sciences (Shanghai, China). The human pancreatic duct epithelial cell line (hTERT-HPNE) was obtained from the American Type Culture Collection (Manassas, VA, USA). The AsPC-1 and BxPC-3 cells were cultured in RPMI 1640 medium (Gibco, Grand Island, NY, USA) supplemented with 10\% fetal bovine serum (Gibco, USA). The PANC-1 and MIA PaCa-2 cells were grown in DMEM (Gibco, USA) containing 10\% fetal bovine serum. The SW1990 cell line 
was maintained in L-15 medium (Gibco, USA) supplemented with $10 \%$ fetal bovine serum. The hTERT-HPNE cell line was cultured in ATCC-suggested complete growth medium (1 V Medium M3, $3 \mathrm{~V}$ glucose-free DMEM, 5\% fetal bovine serum, $10 \mathrm{ng} / \mathrm{ml} \mathrm{EGF,} 5.5 \mathrm{mM}$ D-glucose and $750 \mathrm{ng} / \mathrm{ml}$ puromycin).

\section{Patients and cancer tissues}

This study was approved by the Ethical Committee of Army Medical University, China. All patients provided written informed consent to participate in the study. The study methodologies conformed to the standards set by the Declaration of Helsinki. Eighty pancreatic cancer tissues and ten cancer-adjacent normal tissues were obtained from the Southwest Hospital, Army Medical University (Chongqing, China) between December 2009 and June 2011. All tumor tissues were diagnosed as pancreatic cancer independently by two pathologists. Patients who had received chemotherapy or radiotherapy were excluded. The overall survival (OS) was defined as the interval between the date of definite diagnosis and the date of death or the last follow up. Collection of follow-up data was ceased in July 2016.

\section{Immunohistochemistry (IHC) analysis}

Tissue sections were dewaxed in xylene for $20 \mathrm{~min}$ at $37^{\circ} \mathrm{C}$ and then rehydrated with a series of graded alcohols. To eliminate endogenous peroxidase activity, the sections were incubated with endogenous peroxidase blocking buffer (Beyotime, Shanghai, China). The sections were subsequently treated with a citrate antigen retrieval solution (Beyotime, China) for $20 \mathrm{~min}$ at $98^{\circ} \mathrm{C}$. After washing, the sections were blocked with normal goat serum (Boster, Wuhan, China) for $20 \mathrm{~min}$ at room temperature. The sections were incubated with a mouse monoclonal antibody against human ANLN (1200; Abcam, Cambridge, United Kingdom) overnight at $4{ }^{\circ} \mathrm{C}$. The sections were then washed and incubated with goat anti-mouse secondary antibodies (Boster, Wuhan, China) for $30 \mathrm{~min}$ at room temperature. Finally, the expression levels of ANLN were analyzed according to methods reported [38]. The degree of immunostaining of the sections was defined by the sum of a proportion score and an intensity score. The proportion score was defined as follows: 0 , no positive immunoreactive cells; $1, \leq 10 \%$ positive immunoreactive cells; 2,10 to $50 \%$ positive immunoreactive cells; or $3,>50 \%$ positive immunoreactive cells. The intensity score was defined as follows: 0 , no immunoreactive staining; 1 , weak immunoreactive staining; 2, intermediate immunoreactive staining; or 3, strong immunoreactive staining. The scores were independently evaluated by 2 pathologists.
Small interfering RNA (siRNA), miRNA mimic, miRNA inhibitor, vector transfection and lentiviral particle infection

SiRNAs against ANLN and EZH2 were designed and synthesized by Sesh-biotech (Shanghai, China) (Additional file 1: Table S1). The pCMV3-LASP1 CDS (NM_006148) and pCMV3-EZH2 CDS (NM_004456) expression plasmids were acquired from Sino Biological Inc. (Beijing, China). Mimic control (con), miR-218-5p mimics (miR218-5p), inhibitor control (anti-con) and miR-218-5p inhibitors (anti-miR-218) were obtained from GenePharma (Shanghai, China). For transfection, BxPC-3 and SW1990 cells were cultured in 6-well plates. When the BxPC-3 and SW1990 cells were 80\% confluent, they were transfected with the negative control siRNA (NC), ANLN siRNA (ANLN RNAi) or EZH2 siRNA (EZH2 RNAi) using Lipofectamine 2000 (Invitrogen) according to the manufacturer's instructions. For the rescue experiments, ANLN siRNA together with the pCMV3-LASP1 expression plasmid (LASP1) or pCMV3-EZH2 expression plasmid (EZH2), or miR-218-5p mimic together with the pCMV3LASP1 expression plasmid (LASP1), or EZH2 siRNA together with the miR-218-5p inhibitor were transfected to BxPC-3 and SW1990 cells. To establish the stable ANLNsilencing BxPC-3 cell line, short hairpin RNA (shRNA) oligonucleotide sequences that targeted ANLN were cloned into the pLV-hU6-shRNA-CMV-puromycin lentiviral vector by Sesh-biotech (Shanghai, China). Lentiviral Packaging System was then used for lentivirus packaging. The shRNA sequences are listed in Additional file 1: Table S1. BxPC-3 cells were infected with lentivirus at an MOI (multiplicity of infection) $=15$ and selected with $3 \mu \mathrm{g} / \mathrm{ml}$ puromycin for 15 days.

\section{Western blot analysis}

Cells were harvested, and the total cellular proteins were extracted using RIPA buffer (Sigma-Aldrich, St. Louis, MO, USA) that contained a protease inhibitor cocktail (Roche, Basel, Switzerland). A BCA protein assay kit (Beyotime, China) was subsequently used to quantify the protein concentration. The proteins were then denatured in SDS sample buffers for $10 \mathrm{~min}$ at $100^{\circ} \mathrm{C}$, separated via SDS-PAGE and blotted onto PVDF membranes (BioRad Laboratories, Hercules, CA, USA). The membranes were blocked in Tris-buffered saline that contained 5\% nonfat powdered milk for $1 \mathrm{~h}$. The membranes were subsequently probed with a mouse monoclonal antibody against human ANLN (1:500; Abcam, United Kingdom), mouse monoclonal antibody against human $\beta$-actin (1: 3000; Proteintech, Wuhan, China), rabbit polyclonal antibody against human LASP1 (1:3000; Proteintech, China) or rabbit polyclonal antibody against human EZH2 (1:2000; Proteintech, China) at $4{ }^{\circ} \mathrm{C}$ overnight. After washing, the membranes were incubated with goat 
anti-mouse/rabbit secondary antibodies (1:3000; Santa Cruz Biotechnology, Dallas, TX, USA) at room temperature for $2 \mathrm{~h}$. Finally, the ECL system (Thermo Scientific, Rockford, IL, USA) was used to visualize the protein band signals. The band density was analyzed by Quantity One v4.6.2 (Bio-Rad Laboratories, USA).

\section{Cell proliferation assay}

For the cell proliferation assay, transfected BxPC-3 and SW1990 cells were seeded onto 96-well plates at a final concentration of 2000 cells/well and further incubated for $0,1,2,3$, and $4 \mathrm{~d}$ at $37^{\circ} \mathrm{C}$. Following incubation, the cell viability was measured using a Cell Counting Kit-8 (CCK-8) kit (Beyotime, China) according to the manufacturer's instructions. The CCK- 8 assays were repeated 3 times.

\section{Colony formation assay}

After $48 \mathrm{~h}$ of transfection, BxPC-3 and SW1990 cells were collected, seeded onto 6-well plates at a final concentration of 500 cells/well and cultured for an additional 14 days. The cells were then stained with $0.05 \%$ crystal violet for $20 \mathrm{~min}$. The number of colonies was evaluated via light microscopy. The colony formation assays were performed 3 times.

\section{Cell migration and invasion assay}

The cell migration assay was performed using transwell chambers (BD Biosciences, USA) with a pore size of $8 \mu \mathrm{m}$. The cell invasion assay was performed using Matrigel-coated transwell chambers (BD Biosciences, USA) with a pore size of $8 \mu \mathrm{m}$. At $48 \mathrm{~h}$ after transfection, BxPC-3 and SW1990 cells were seeded onto the upper chambers at a final concentration of $5 \times 10^{4}$ cells/well and cultured in $100 \mu \mathrm{l}$ of serum-free medium. The lower chambers contained $700 \mu \mathrm{l}$ of medium with $10 \%$ FBS. After $48 \mathrm{~h}$ of incubation, the cells migrated or invaded through the filter into the lower side of the chamber were fixed and stained with crystal violet for $30 \mathrm{~min}$. The number of cells was counted under a microscope. Each experiment was performed in triplicate.

\section{In vivo xenograft tumor models}

This study was approved by the Ethical Committee of Army Medical University, China. The stable ANLN-silenced BxPC- 3 cells (LV-ANLN shRNA, $1 \times 10^{6}$ cells in $100 \mu \mathrm{l}$ of sterilized PBS) and the stable scramble control BxPC-3 cells (LV-NC, $1 \times 10^{6}$ cells in $100 \mu$ of sterilized $\mathrm{PBS}$ ) were injected into the right and left dorsal flanks of 4-week-old BALB/c male nude mice (Animal Center of the Chinese Academy of Science, Shanghai, China), respectively. Next, all mice were raised in a pathogen-free condition. The lengths and widths of the tumors were measured every week, and the tumor volume was calculated as follows: tumor volume $=\left(\right.$ length $\times$ width $\left.^{2}\right) /$ 2. At 5 weeks after injection, all mice were euthanized, and their tumors were dissected. The tumors were subjected to IHC staining to analyze the expression levels of ANLN, EZH2 and LASP1 with primary antibodies against ANLN (1:200; Abcam, Cambridge, United Kingdom), EZH2 (1:200; Abcam, Cambridge, United Kingdom) and LASP1 (1:200; Proteintech, China).

\section{Microarray analysis}

BxPC-3 cells were transfected with NC or ANLN RNAi and collected after 3 days, and three biological replicates were utilized. Total RNA was extracted using TRIzol reagent (Invitrogen, Grand Island, NY, USA). The RNA quality was determined by a spectrophotometer at 260 and $280 \mathrm{~nm}$. The RNA integrity was evaluated by electrophoresis ( $1 \%$ formaldehyde denaturing gel). The RNA was subsequently synthesized into cDNA, and converted into cRNA. Labeled cRNA was hybridized to the Affymetrix Gene Chip Human Gene 1.0 ST Array (Affymetrix, Santa Clara, CA, USA). Expression Console and Transcriptome Analysis Console v3.0 (Affymetrix, USA) were used to analyze differentially expressed genes. Gene ontology (GO) annotation analysis was performed using DAVID Bioinformatics Resources 6.8 (https://david.ncifcrf.gov/).

\section{Quantitative reverse transcription polymerase chain reaction ( $q R T-P C R$ )}

Total RNA was isolated from cells using TRIzol reagent (Invitrogen, USA), according to manufacturer's instructions. A spectrophotometer was used to determine the RNA quality and quantity. RNA was then reverse-transcribed into cDNA using M-MLV Reverse Transcriptase (TaKaRa, Dalian, China) and BulgeLoop ${ }^{\text {TM }}$ specific RT-primers and random primers for mRNA. The gene expression levels were analyzed using a SYBR Premix Ex Taq kit (TaKaRa, China). The relative expression of gene was calculated using the $2^{-\Delta \Delta C t}$ method. $\beta$-actin or U6 was used as an internal control. This experiment was performed with three biological replicates. All primer sequences are listed in Additional file 1: Table S1.

\section{Luciferase reporter assay}

The partial wild-type sequence of the LASP1 3' -untranslated region (UTR) (2262 bp) containing the three putative miR-218-5p binding sites (Site1:686-692, Site2: 1587-1593 and Site3 2080-2087) or the sequences having mutations of the miR-218-5p putative binding sites in LASP1 3'UTR were cloned into the downstream of the luciferase gene in the psiCHECK-2 vector (Promega, Madison, WI, USA).

BxPC-3 and SW1990 cells were cotransfected with the LASP1 3'UTR luciferase expression plasmid and the miR-218-5p mimic or con using Lipofectamine 2000 
(Invitrogen). After $48 \mathrm{~h}$ of transfection, a dual-luciferase reporter assay system (Promega, Madison, WI, USA) was used to determine the luciferase activity. The firefly luciferase activity was normalized to Renilla activity.

\section{Statistical analysis}

Data from Western blot, cell proliferation, colony formation, cell migration, cell invasion, qRT-PCR, Luciferase reporter and in vivo tumor growth assays were analyzed using SPSS17.0 statistical software (IBM Corporation, Armonk, NY, USA) and presented as an average of biological replicates (mean \pm S.D.). Student's t-test or one-way ANOVA was used to evaluate the differences. Associations between ANLN expression and the clinicopathologic parameters were determined by the non-parametric Pearson Chi-Square test. The survival rates for each variable were analyzed using the Kaplan-Meier method. Moreover, logrank statistics were used to estimate the equivalences of the survival curves. The parameters with statistical significance in the univariate survival analysis were subjected to further evaluation via multivariate survival analysis. $P$ values $<0.05$ were considered to be statistically significant.

\section{Results}

ANLN expression was upregulated in pancreatic cancer tissues and cell lines

According to the GENT database, ANLN expression was significantly upregulated in 174 pancreatic cancer tissues compared with that in 62 normal tissues $(P<$ 0.001, Fig. 1a) [39]. In addition, results from cBioportal for Cancer Genomic (www.cbioportal.org/) showed that the mRNA upregulation of the ANLN gene accounted for most of the alterations (QCMG, Nature 2016; TCGA, Pancancer Atlas; TCGA, Provisional; UTSW, Nat Commun) (Fig. 1b) [40, 41]. Moreover, fewer ANLN shallow deletion and high-level ANLN gene amplification (amplification) and more ANLN diploid and low-level ANLN gene amplification (gain) were observed in two pancreatic cancer datasets (Pancreatic Adenocarcinoma-TCGA, PanCancer Atlas and Pancreatic Adenocarcinoma-TCGA, Provisional) (Fig. 1c). The ANLN mRNA expression in the pancreatic cancer samples with low-level ANLN gene amplification (gain) was markedly increased compared with that in the pancreatic cancer samples with ANLN diploids (Fig. 1c). In addition, Kaplan-Meier plots summarizing the result from the Human Protein Atlas (www.proteinatlas. org/) showed that the upregulation of ANLN was significantly correlated with worse overall survival $(P=0.000002$, Fig. 1d). Consistent with these publicly available data, our results also showed significantly upregulated ANLN expression in pancreatic cancer tissues compared with that in their matched adjacent normal pancreatic tissues (Fig. 1e). We subsequently analyzed the association between the ANLN expression and clinicopathological features in 80 pancreatic cancer samples. We found that ANLN upregulation was significantly correlated with the tumor size $(P=0.002)$, tumor differentiation $(P=0.027)$, TNM stage $(P<0.001)$, lymph node metastasis $(P=0.004)$ and distant metastasis $(P=0.005)$ (Table 1$)$. In addition, univariate analysis indicated that the TNM stage, lymph node metastasis and upregulated ANLN expression were associated with overall survival $(P=0.002, P=$ 0.016 and $P=0.034$, respectively) (Table 2). Multivariate analysis showed that the TNM stage, lymph node metastasis and upregulated ANLN expression were independent prognostic factors for overall survival $(P=$ $0.001, P=0.021$ and $P=0.002$, respectively) (Table 2). Kaplan-Meier analysis showed that upregulated ANLN expression was significantly associated with shorter survival for patients with pancreatic cancer $(P=0.001$, Fig. 1e). Moreover, we analyzed the ANLN protein expression in one normal human pancreatic duct epithelial cell line (hTERT-HPNE) and five pancreatic cancer cell lines (AsPC-1, PANC-1, BxPC-3, MIA PaCa-2 and SW1990). Our results showed that ANLN protein expression was significantly increased in the five pancreatic cancer cell lines compared with that in the hTERT-HPNE cell line (Fig. 1f).

\section{ANLN downregulation inhibited pancreatic cancer tumorigenesis in vitro and in vivo}

To determine the function of ANLN in pancreatic cancer, we conducted siRNA-mediated gene silencing. As shown in Fig. 2a, ANLN expression was significantly reduced in the BxPC-3 and SW1990 cell lines after transfection with ANLN siRNA (ANLN RNAi) compared with that in the negative control group $(\mathrm{NC})(P<0.01)$. After confirming the suppressive effect of ANLN siRNA by Western blot, we performed CCK- 8 assays and showed that ANLN downregulation significantly inhibited BxPC-3 and SW1990 cell proliferation when compared with that in the NC group (Fig. 2b). In the colony formation assay, the colony numbers were significantly decreased in cells transfected with ANLN siRNA compared with those transfected with the control (Fig. 2c). The migration and invasion assay indicated that knockdown of ANLN significantly suppressed cell migration and invasion (Fig. 2d and e). Moreover, we established the stable ANLN silencing BxPC-3 cell line using lentiviral vectors with puromycin. The knockdown effect of ANLN-silencing lentiviral vectors was confirmed by Western blot. The ANLN-silencing lentiviral vectors (LVANLN shRNA) clearly inhibited the ANLN protein expression in BxPC-3 cells compared with that resulting from the control lentiviral vectors (LV-NC) (Fig. 2f). Moreover, the xenograft tumor volumes of the LV-ANLN shRNA group were obviously reduced when compared with those of the LV-NC group $(P<0.01$, Fig. $2 \mathrm{~g})$. 


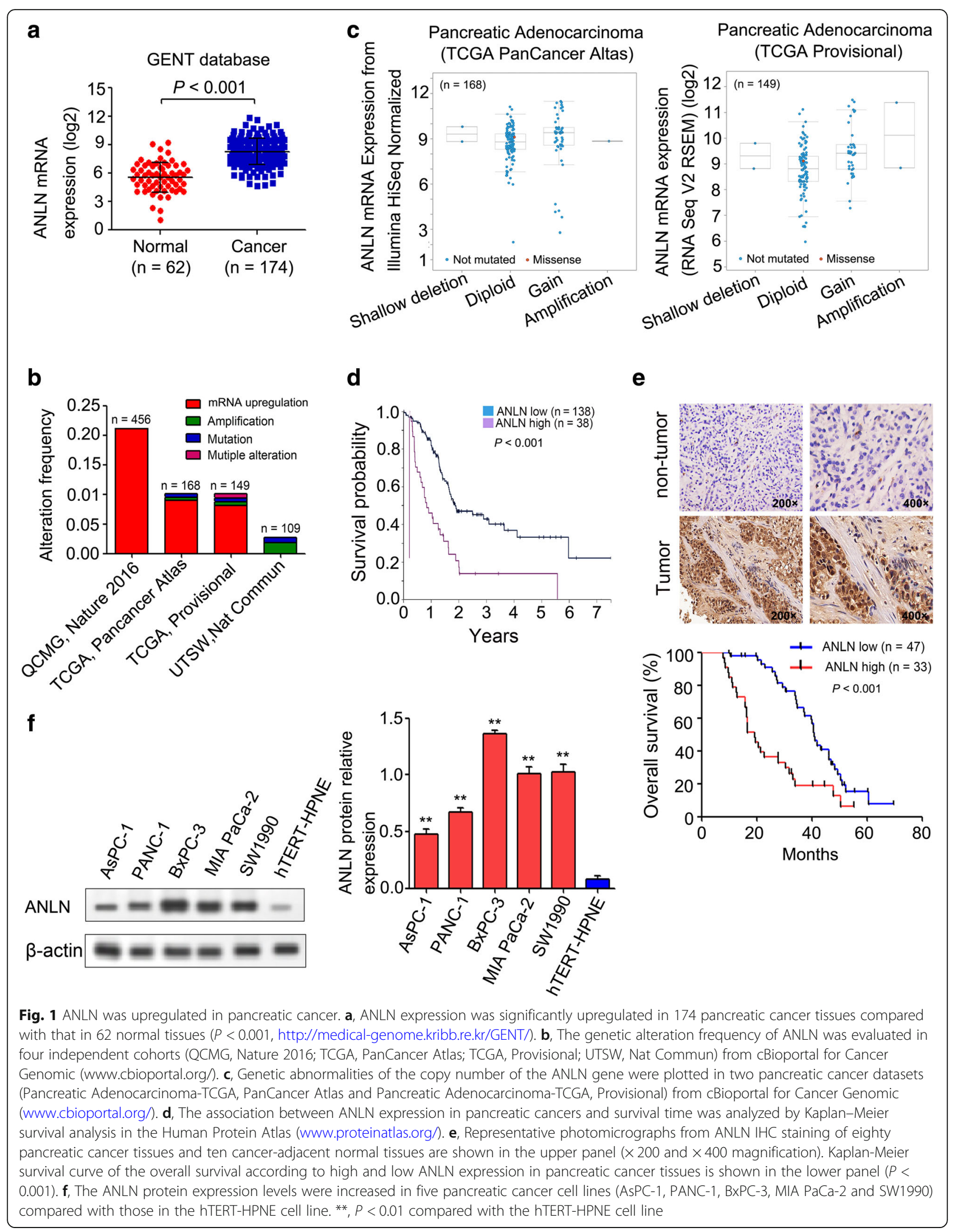


Table 1 Correlations between ANLN expression and clinicopathological characteristics of patients with pancreatic cancer

\begin{tabular}{|c|c|c|c|c|}
\hline \multirow[t]{2}{*}{ Variable } & \multirow[t]{2}{*}{ All cases } & \multicolumn{2}{|c|}{ ANLN } & \multirow[t]{2}{*}{$P$ value } \\
\hline & & Low expression & High expression & \\
\hline \multicolumn{4}{|l|}{$\overline{\text { Age }}$} & \multirow[t]{3}{*}{0.429} \\
\hline$\leq 60$ & 37 & 20 & 17 & \\
\hline$>60$ & 43 & 27 & 16 & \\
\hline \multicolumn{5}{|l|}{ Gender } \\
\hline Male & 46 & 26 & 20 & \multirow[t]{2}{*}{0.638} \\
\hline Female & 34 & 21 & 13 & \\
\hline \multicolumn{5}{|c|}{ Tumor size $(\mathrm{cm})$} \\
\hline$<2$ & 41 & 31 & 10 & \multirow[t]{2}{*}{$0.002^{* *}$} \\
\hline$\geq 2$ & 39 & 16 & 23 & \\
\hline \multicolumn{5}{|c|}{ Tumor differentiation } \\
\hline Well & 36 & 26 & 10 & \multirow[t]{2}{*}{$0.027^{*}$} \\
\hline Poor & 44 & 21 & 23 & \\
\hline \multicolumn{5}{|l|}{ TNM stage } \\
\hline $\mid+\|$ & 25 & 22 & 3 & \multirow[t]{2}{*}{$0.000^{* *}$} \\
\hline$I I I+I V$ & 55 & 25 & 30 & \\
\hline \multicolumn{5}{|c|}{ Lymph node metastasis } \\
\hline Negative & 49 & 35 & 14 & \multirow[t]{2}{*}{$0.004^{* *}$} \\
\hline Positive & 31 & 12 & 19 & \\
\hline \multicolumn{5}{|c|}{ Distant metastasis } \\
\hline MO & 53 & 37 & 16 & \multirow[t]{2}{*}{$0.005^{* *}$} \\
\hline M1 & 27 & 10 & 17 & \\
\hline
\end{tabular}

*Statistically significant $(P<0.05), *$ * Statistically significant $(P<0.01)$

\section{Downregulation of ANLN in BxPC-3 cells exerted strong} effects on gene expression

To explore the potential mechanisms of ANLN in regulating pancreatic cancer progression, gene microarray analysis was performed using RNA isolated from BxPC-3 cells transfected with NC or ANLN RNAi. A total of 1926 genes were downregulated with fold changes $<-2.5$
$(P<0.05)$, while 631 genes were upregulated with fold changes $>2.5(P<0.05)$ (Fig. $3 \mathrm{a}$ and $\mathrm{b})$. The downregulated and upregulated genes were subsequently submitted to gene ontology (GO) annotation analysis using the DAVID Bioinformatics Resources 6.8 (https://david.ncifcrf. gov/). The Go terms representing biological processes indicated that these differentially expressed genes were highly enriched in cell-cell adhesion and cell cycle-related biological processes (Additional file 2: Table S2, Fig. 3c). The GO terms representing cellular compartment and molecular functions were shown in Additional file 3: Table S3 and Additional file 4: Table S4. Interestingly, cell-cell adhesion-related genes are largely involved in regulating cell growth, proliferation and motility [42, 43]. Based on GO analysis, 54 cell-cell adhesion-related genes were shown in Fig. 3d. According to the clustering results shown in Fig. 3d, eleven candidate genes were selected. To validate the microarray results, qRT-PCR was used to determine the expression of the eleven genes. Similar to the microarray results, the expression of all eleven genes in BxPC-3 cells transfected with ANLN RNAi was significantly decreased compared with that in the NC group (Fig. 3e).

\section{Upregulation of LASP1 contributed to the aggressive properties regulated by ANLN}

Among the eleven candidate genes selected according to the clustering results, LASP1, RAB11B, RUVBL1 and MYO1B are upregulated in human malignant tumors and contribute to cancer progression [20, 44-46]. Based on the GENT database, we showed that both LASP1 and RUVBL1 gene expression were significantly upregulated in pancreatic cancer tissues compared with that in normal pancreatic tissues, while RAB11B was not changed obviously, and MYO1B was significantly downregulated (Additional file 5: Figure S1A). In addition, both LASP1 and RUVBL1 expression were positively correlated with ANLN expression (Additional file 5: Figure S1B). Further investigation showed that LASP1 restoration partially

Table 2 Cox proportional hazard models for prognostic factors

\begin{tabular}{|c|c|c|c|c|}
\hline & \multicolumn{2}{|l|}{ Univariate analysis } & \multicolumn{2}{|l|}{ Multivariate analysis } \\
\hline & $\mathrm{HR}(95 \% \mathrm{Cl})$ & $P$ value & $\mathrm{HR}(95 \% \mathrm{Cl})$ & $P$ value \\
\hline Age $(>60$ vs. $\leq 60)$ & $1.371(0.749-2.510)$ & 0.306 & & \\
\hline Gender (famale vs. male) & $0.874(0.491-1.557)$ & 0.648 & & \\
\hline Tumor size $(\geq 2$ vs. $<2)$ & $1.502(0.836-2.699)$ & 0.173 & & \\
\hline Tumor differentiation (Poor vs. Well) & $1.287(0.738-2.244)$ & 0.375 & & \\
\hline TNM stage (III + IV vs. I + II) & $3.013(1.495-6.073)$ & $0.002^{* *}$ & $3.043(1.542-6.004)$ & $0.001^{* *}$ \\
\hline Lymph node metastasis (Positive vs. Negative) & $2.109(1.151-3.864)$ & $0.016^{*}$ & $1.873(1.100-3.188)$ & $0.021^{*}$ \\
\hline Distant metastasis (M1 vs. M0) & $1.244(0.679-2.280)$ & 0.479 & & \\
\hline ANLN expression (high vs. low) & $1.923(1.051-3.521)$ & $0.034^{*}$ & $2.352(1.369-4.039)$ & $0.002^{* *}$ \\
\hline
\end{tabular}

*Statistically significant $(P<0.05),{ }^{*}$ Statistically significant $(P<0.01)$ 


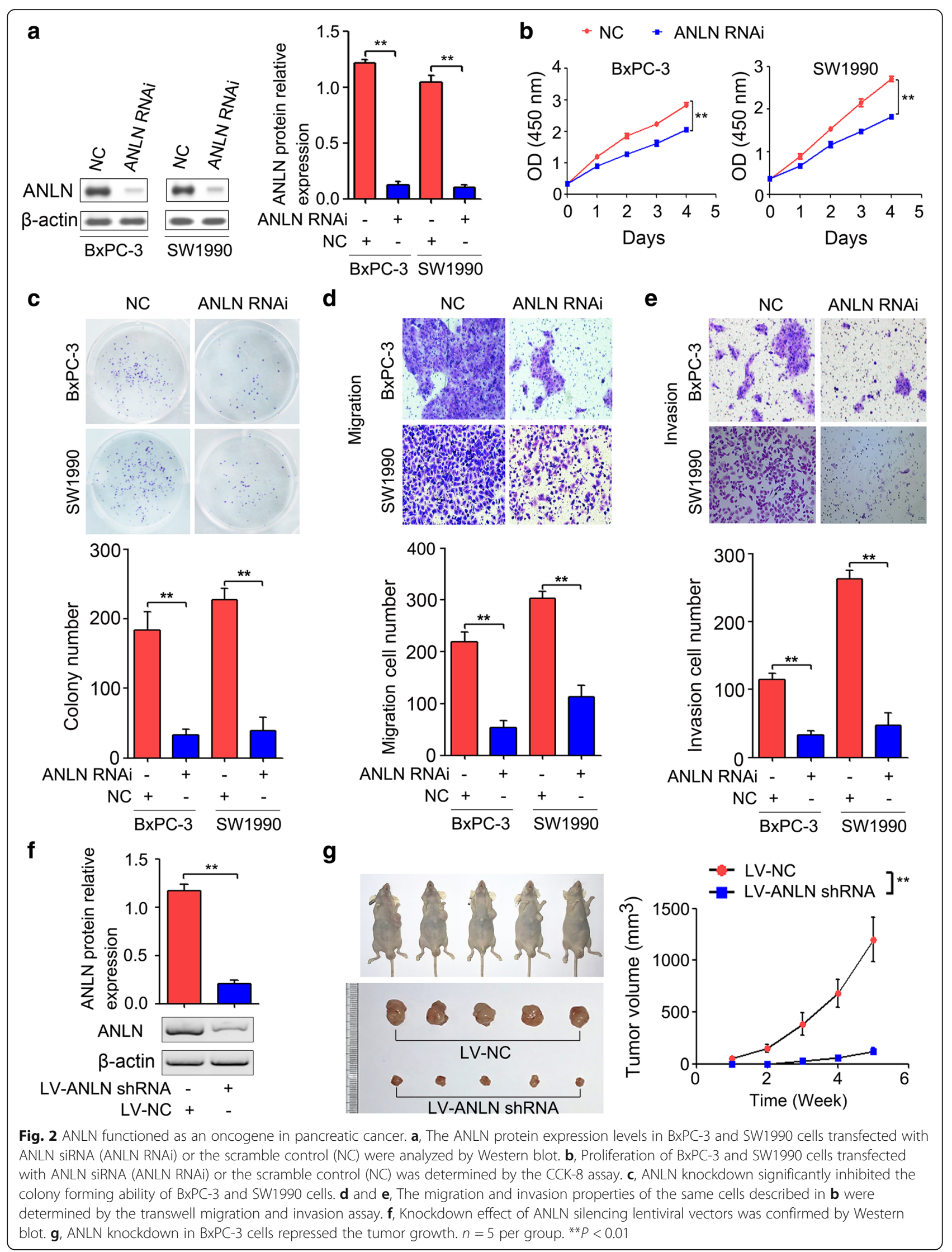



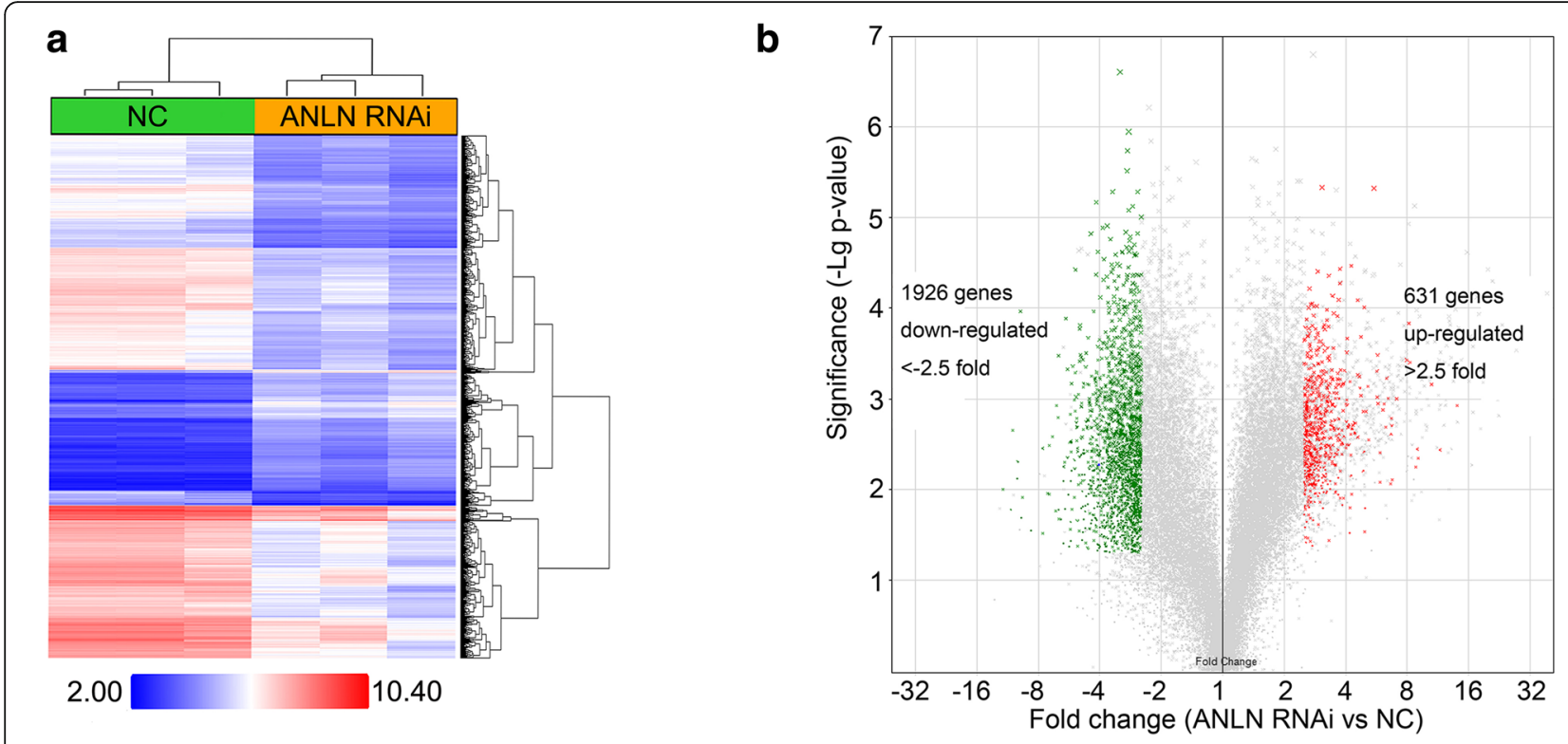

\section{Gene ontology Biological process}

-Lg p-value

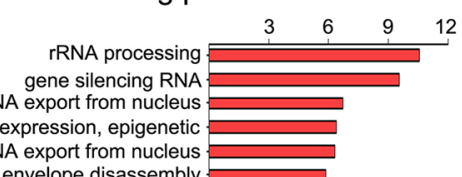

positive regulation of gene expression, epigenetic mRNA export from nucleus mitotic nuclear envelope disassembly DNA replication initation translation regulation of glucose transport protein sumoylation
translation initation DNA replication-dependent nucleosome assembly chromatin silencing at rDNA $\mathrm{G} 1 / \mathrm{S}$ transition of mitotic cell cycle
positive regulation of ubiquitin-protein ligase activity involved in regulation mitotic cell cycle transition

in heterotetramerization mitotic nuclear division
vesicle docking involved in exocytosis anaphase-promoting complex-dependent catabolic process nucleosome assembly negative regulation of gene expression, epigenetic negative regulation of ubiquitin-protein ligase activity DNA unwinding involved in DNA replication.

e

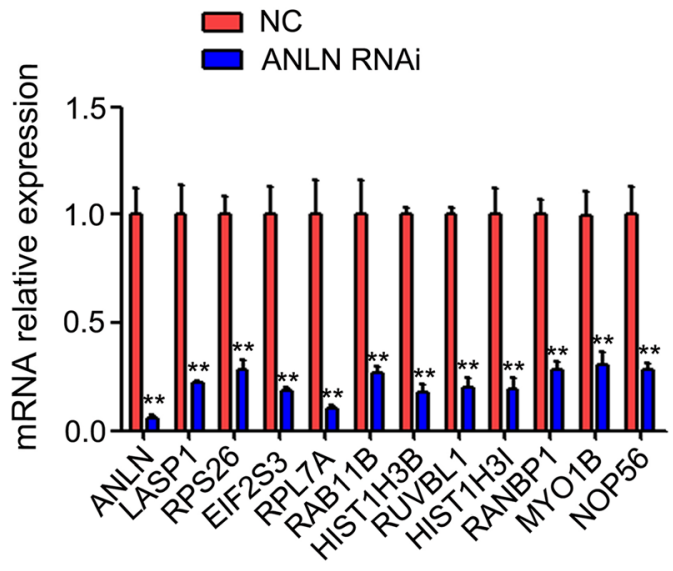

d

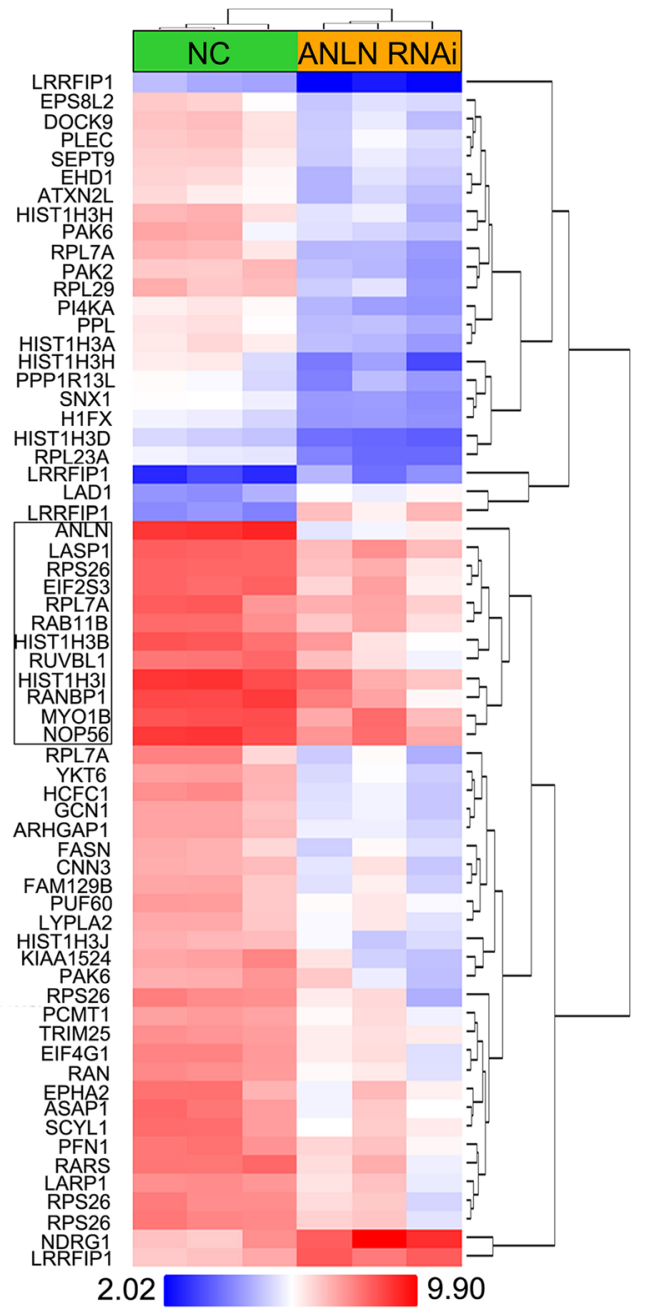

Fig. 3 (See legend on next page.) 
(See figure on previous page.)

Fig. 3 Global changes in gene expression in BxPC-3 cells transfected with ANLN siRNA. a, The heat map of the differentially expressed genes with a fold change of greater than 2.5 or less than -2.5 in ANLN RNAi relative to NC. $\mathbf{b}$, A volcano plot showing the differentially expressed genes with a fold change of greater than 2.5 or less than - 2.5 in ANLN RNAi relative to NC. c, The top twenty-five regulated Gene ontology (GO) biological process terms. $\mathbf{d}$, The heat map of 54 cell-cell adhesion-related genes. $\mathbf{e}$, The selected candidate genes from microarray experiments were confirmed by qRT-PCR after ANLN RNAi transfection in BxPC-3 cells. ${ }^{*} P<0.01$ compared with the NC group

reversed the effects of ANLN knockdown on pancreatic cancer cell proliferation (Additional file 5: Figure S1C). However, RUVBL1 restoration did not reverse the effect of ANLN downregulation on pancreatic cancer cell proliferation (Additional file 5: Figure S1C). Thus, ANLN may promote pancreatic cancer cell progression by regulating LASP1. To confirm this hypothesis, we rescued LASP1 expression using LASP1 overexpression plasmid vectors in the cells transfected with ANLN RNAi. As shown in Fig. 4a, the LASP1 protein expression in ANLN RNAitransfected cells was restored by the LASP1 plasmid. In addition, the CCK- 8 and colony formation assays indicated that the suppressive effects of ANLN knockdown on pancreatic cancer cell growth were restored by LASP1 re-expression (Fig. 4b and c). Moreover, LASP1 re-expression partially reversed the effect of ANLN knockdown on pancreatic cancer cell migration and invasion (Fig. 4d and e).

\section{LASP1 is a crucial target of miR-218-5p in pancreatic cancer}

Previous reports have shown that microRNAs (miRNAs) play an important role in cancer initiation and tumor progression by directly regulating target genes expression [47]. Here, we found that a total of 49 miRNA precursors showed significant changes in gene expression profiles; 46 miRNA precursors were upregulated, and 3 miRNA precursors were downregulated (Additional file 6: Figure S2A). By combining the gene expression profiles and Targetscan (http://www.targetscan.org/), three miRNAs (miR-145-5p, miR-218-5p and miR-9-5p) were found both upregulated in ANLN downregulated BxPC-3 cells and contained binding sites of the 3'UTR of LASP1 (Additional file 6: Figure S2B). To determine the effect of miR-145-5p, miR-218-5p and miR-9-5p on LASP1 expression, miR-145-5p, miR-218-5p and miR-9-5p mimics were transfected into $\mathrm{BxPC}-3$ cells, respectively. We found that the expression of miR-145-5p, miR-218$5 p$ and miR-9-5p in transfected cells was significantly upregulated (Additional file 6: Figure S2C). Additional qRT-PCR showed that only miR-218-5p upregulation significantly repressed LASP1 mRNA expression in BxPC-3 cells (Additional file 6: Figure S2D). To further confirm the effect of miR-218-5p on LASP1 expression, the mimic control (con), miR-218-5p mimics (miR-218$5 p)$ were transfected into BxPC-3 and SW1990 cells, respectively. As shown in Fig. 5a, the expression of miR-
$218-5 \mathrm{p}$ in transfected cells was significantly upregulated. Moreover, miR-218-5p upregulation significantly suppressed LASP1 protein expression in BxPC-3 and SW1990 cells (Fig. 5b). To determine whether miR218-5p directly targets the 3'UTR of LASP1, the luciferase reporter vectors containing the 3'UTR of LASP1 (wt-LASP1) or its mutant version were constructed (Fig. 5c). We found that the luciferase activity of miR218-5p-transfected cells was significantly inhibited compared with that of the con-transfected cells. In addition, site-directed mutagenesis of the miRNA binding sequences in the 3'UTR of LASP1 showed a slightly different trend. The conserved miRNA binding site (Site3) appeared to be slightly more effective in miR218b-5p-induced luciferase activity repression because mutation of this site significantly reversed a decrease in the luciferase signal (Fig. 5d). However, the miR-218$5 p$-mediated repression of luciferase activity was abolished by mutating the three binding sequences (triple mutation) of the LASP1 3'UTR (Fig. 5d). To further determine the role of LASP1 in the suppressive effects of miR-218-5p on pancreatic cancer progression, we rescued the expression of LASP1 by LASP1 overexpression plasmid vectors in the cells transfected with miR218-5p. As shown in Fig. 5e, LASP1 protein expression in miR-218-5p-transfected cells was restored by LASP1 plasmid. In addition, CCK- 8 analysis and colony formation assay revealed that the suppressive effects of miR218-5p upregulation in pancreatic cancer cell growth were partially restored by LASP1 re-expression (Fig. $5 \mathrm{f}$ and g). Moreover, LASP1 re-expression partially reversed the inhibition of cell migration and invasion caused by miR-218-5p upregulation (Fig. $5 \mathrm{~h}$ and i).

miR-218-5p was responsible for the ANLN-induced LASP1 expression and pancreatic cancer cell growth, migration and invasion

In this study, we showed that miR-218-5p upregulation inhibited pancreatic cancer cell growth, migration and invasion by directly regulating LASP1 expression. Moreover, ANLN knockdown significantly induced the expression of miR-218-5p. Thus, ANLN may regulate LASP1 expression and pancreatic cancer progression by miR-218-5p. To determine whether miR-218-5p was involved in ANLN-induced LASP1 expression and pancreatic cancer cell growth, migration and invasion, miR218-5p inhibitor (anti-miR-218) was used to reverse the 


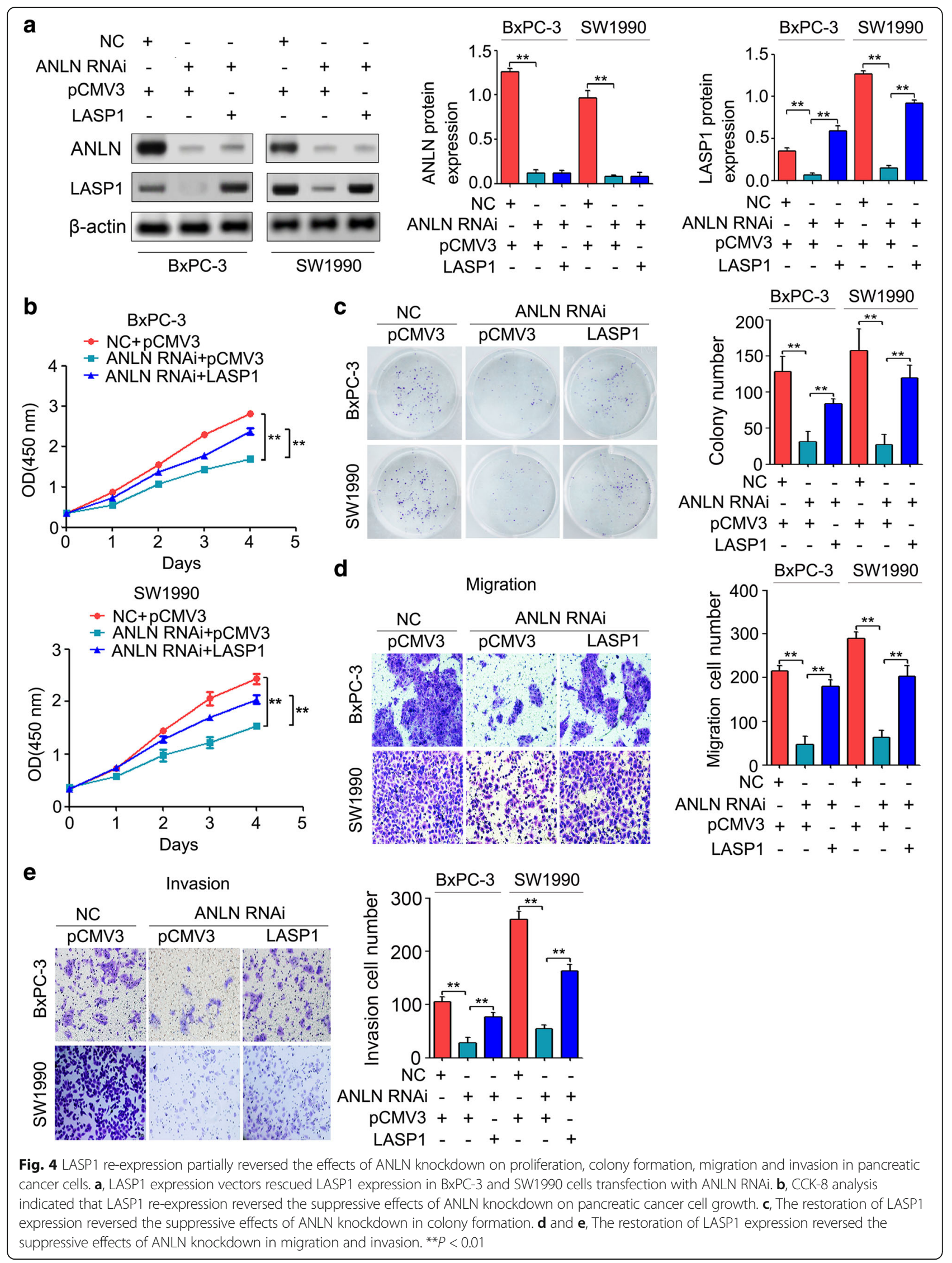




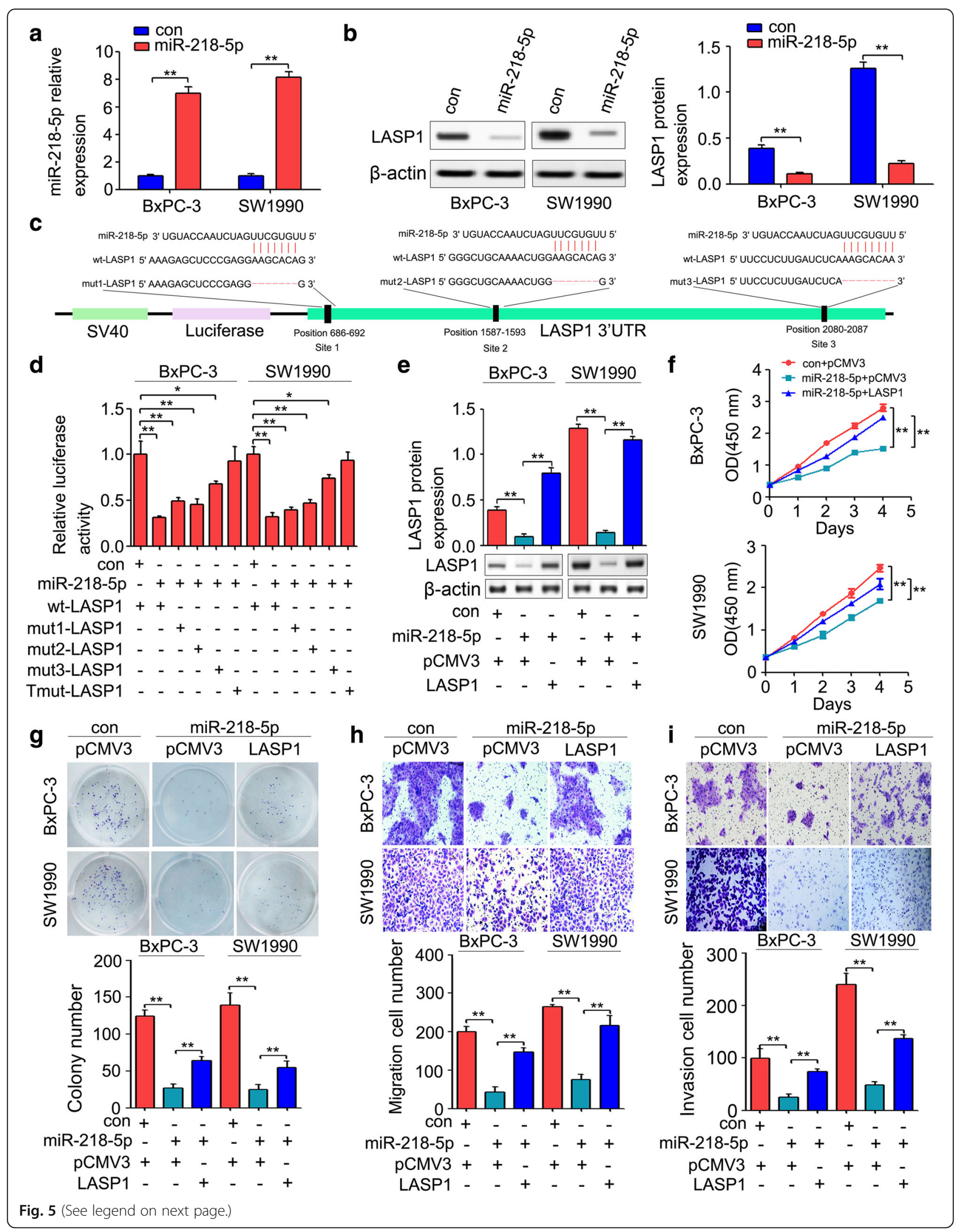


(See figure on previous page.)

Fig. 5 MiR-218-5p inhibited pancreatic cancer cell progression by directly regulating LASP1. a, The expression levels of miR-218-5p were detected by qRT-PCR in BXPC-3 and SW1990 cells transfected with the miR-218-5p mimic (miR-218-5p) or mimic control (con). U6 was used as a loading control. b, The expression levels of LASP1 protein in BxPC-3 and SW1990 cells transfected with the miR-218-5p mimic (miR-218-5p) or mimic control (con) were analyzed by Western blot. c, Representation of the seed pairing between miR-218-5p and LASP1 3'UTR. Three mutations (mut1-LASP1, mut2-LASP1 and mut3-LASP1) were generated for luciferase assay in the sequence complementary to the miR-218-5p target binding region. d, BXPC-3 and SW1990 cells were co-transfected with either miR-218-5p mimic (miR-218-5p) or mimic control (con) and the wildtype or mutant LASP1 3'UTR luciferase reporter vector for $48 \mathrm{~h}$. The relative luciferase activity was determined by the dual-luciferase assay. Renilla luciferase activity was used as a loading control. e, Efficiency of LASP1 re-expression was determined by Western blot. f, CCK-8 analysis revealed that LASP1 re-expression partly reversed the growth repression of miR-218-5p on pancreatic cancer cells. $\mathbf{g}$, The restoration of LASP1 expression reversed the suppressive effects of miR-218-5p in colony formation. $\mathbf{h}$ and $\mathbf{I}$, Ectopic expression of LASP1 reversed the suppressive effects of miR218-5 $p$ in migration and invasion. ${ }^{*} P<0.05,{ }^{*} P<0.01$

expression of miR-218-5p upregulation caused by ANLN knockdown. As shown in Fig. 6a, anti-miR-218 obviously reversed the ANLN knockdown-induced miR-218-5p expression. In addition, the LASP1 protein levels were restored in the cells cotransfected with ANLN RNAi and anti-miR-218 compared with the protein levels in the cells cotransfected with ANLN RNAi and inhibitor control (anti-con) (Fig. 6b). In functional assays, miR-218$5 \mathrm{p}$ knockdown in pancreatic cancer cells transfected with ANLN RNAi rescued the inhibition of cell proliferation, colony formation, cell migration and cell invasion caused by ANLN knockdown (Fig. 6c-f). Collectively, these results demonstrated that ANLN promotes pancreatic cancer cell growth, migration and invasion by regulating miR-218-5p/LASP1 signaling axis.

\section{EZH2 was involved in ANLN-induced pancreatic cancer cell growth, migration and invasion by mediating miR- 218-5p/LASP1 signaling axis}

In pancreatic cancer, EZH2 is upregulated and induces pancreatic cancer cell proliferation, cell migration and cell invasion in vitro and tumor growth and metastasis in vivo by inhibiting the expression of miR-218-5p [37]. In this study, the result of gene expression profiles showed that ANLN knockdown significantly inhibited the expression of EZH2 (Additional file 7: Figure S3A). To further confirm the effect of ANLN knockdown on EZH2, qRT-PCR and Western blot analysis was performed. Our results showed that ANLN knockdown significantly repressed the expression of EZH2 mRNA and protein (Additional file 7: Figure S3B and C). Therefore, ANLN may promote pancreatic cancer cell progression and miR-218-5p/LASP1 signaling axis by mediating EZH2. To determine whether EZH2 regulates LASP1, we transfected the BxPC-3 and SW1990 cells with the negative control siRNA (NC) or EZH2 siRNA (EZH2 RNAi). As shown in Fig. 7a, EZH2 downregulation significantly inhibited the expression of LASP1 protein. In addition, to further confirm whether EZH2 downregulation could inhibit LASP1 expression by inducing miR-218-5p expression, miR-218-5p inhibitor (antimiR-218) was used to reverse the EZH2 knockdown- induced miR-218-5p expression. As shown in Fig. 7b, anti-miR-218 obviously reversed the EZH2 knockdowninduced miR-218-5p expression. Moreover, the LASP1 protein levels were restored in the cells cotransfected with EZH2 RNAi and anti-miR-218 compared with the protein levels in the cells cotransfected with EZH2 RNAi and inhibitor control (anti-con) (Fig. 7c). To further determine whether EZH2 was involved in ANLN-induced LASP1 expression and the inhibition of miR-218-5p, EZH2 expression plasmid vectors were used to rescue the inhibition of EZH2 caused by ANLN knockdown. As shown in Fig. 7d, the ectopic expression of EZH2 reversed the upregulation of miR-218-5p expression caused by ANLN knockdown. In addition, EZH2 re-expression reversed the inhibition of LASP1 caused by ANLN knockdown (Fig. 7e). In functional assays, EZH2 restoration in pancreatic cancer cells transfected with ANLN RNAi rescued the inhibition of cell proliferation, colony formation, cell migration and cell invasion caused by ANLN knockdown (Fig. 7f-h).

\section{Downregulation of ANLN contributed to the inhibition of EZH2 and LASP1 in vivo}

To confirm the effect of ANLN knockdown on the expression of EZH2 and LASP1, we detected the levels of ANLN, EZH2 and LASP1 expression in tissues from in vivo xenograft tumor models established with the stable ANLN-silenced BxPC-3 cells or the stable scramble control BxPC-3 cells by IHC and Western blot. IHC staining showed that the levels of EZH2 and LASP1 expression were repressed after ANLN knockdown (Fig. 8a). Consistent with these findings, Western blot results also showed that the levels of EZH2 and LASP1 expression were significantly inhibited in the LV-ANLN-shRNA group when compared to the LV-NC group (Fig. 8b). Taken together, ANLN may promote pancreatic cancer cell growth, migration and invasion by regulating EZH2/miR-218-5p/LASP1 signaling axis (Fig. 9).

\section{Discussion}

The present study demonstrated that ANLN was upregulated in pancreatic cancer, and upregulated ANLN was 


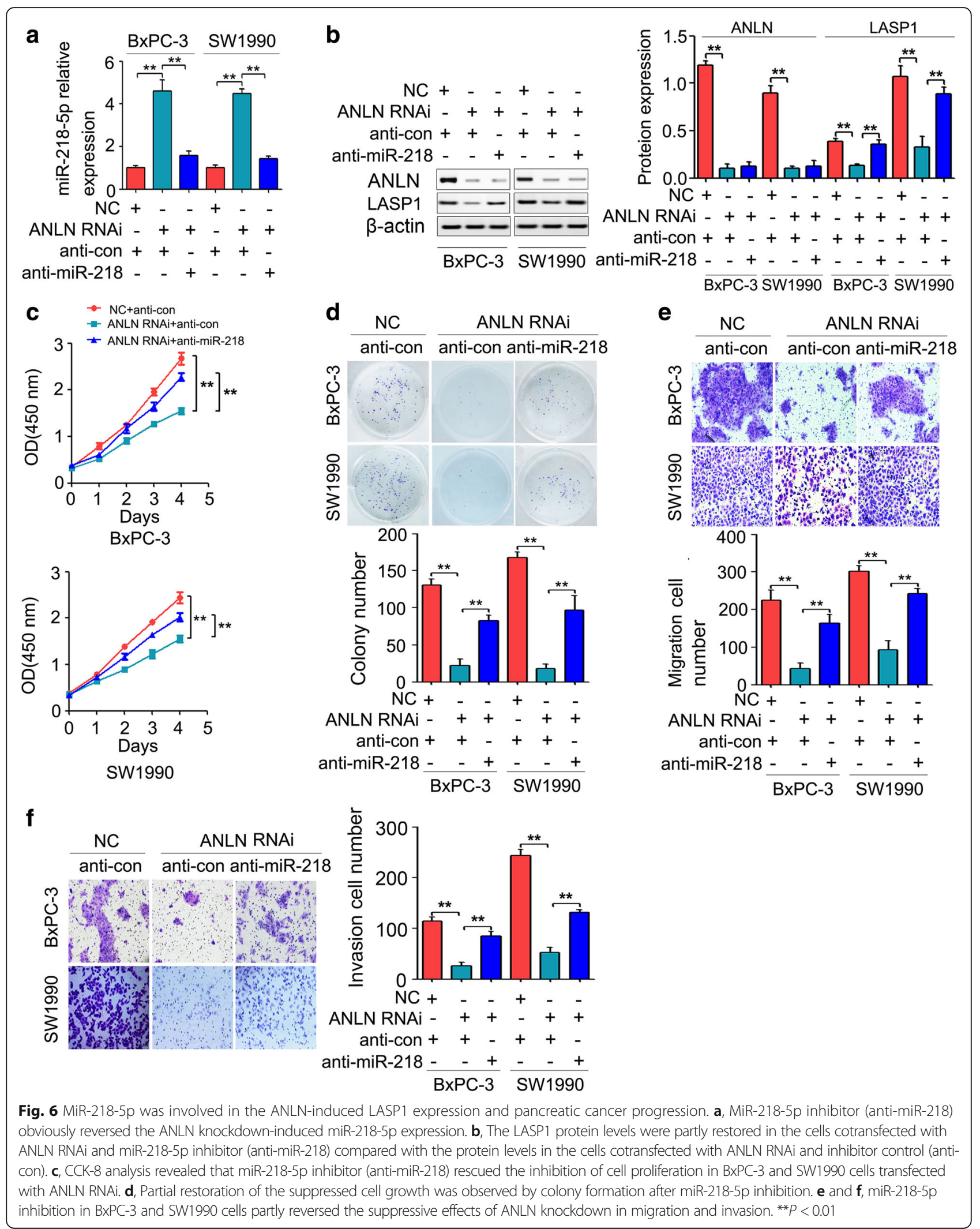




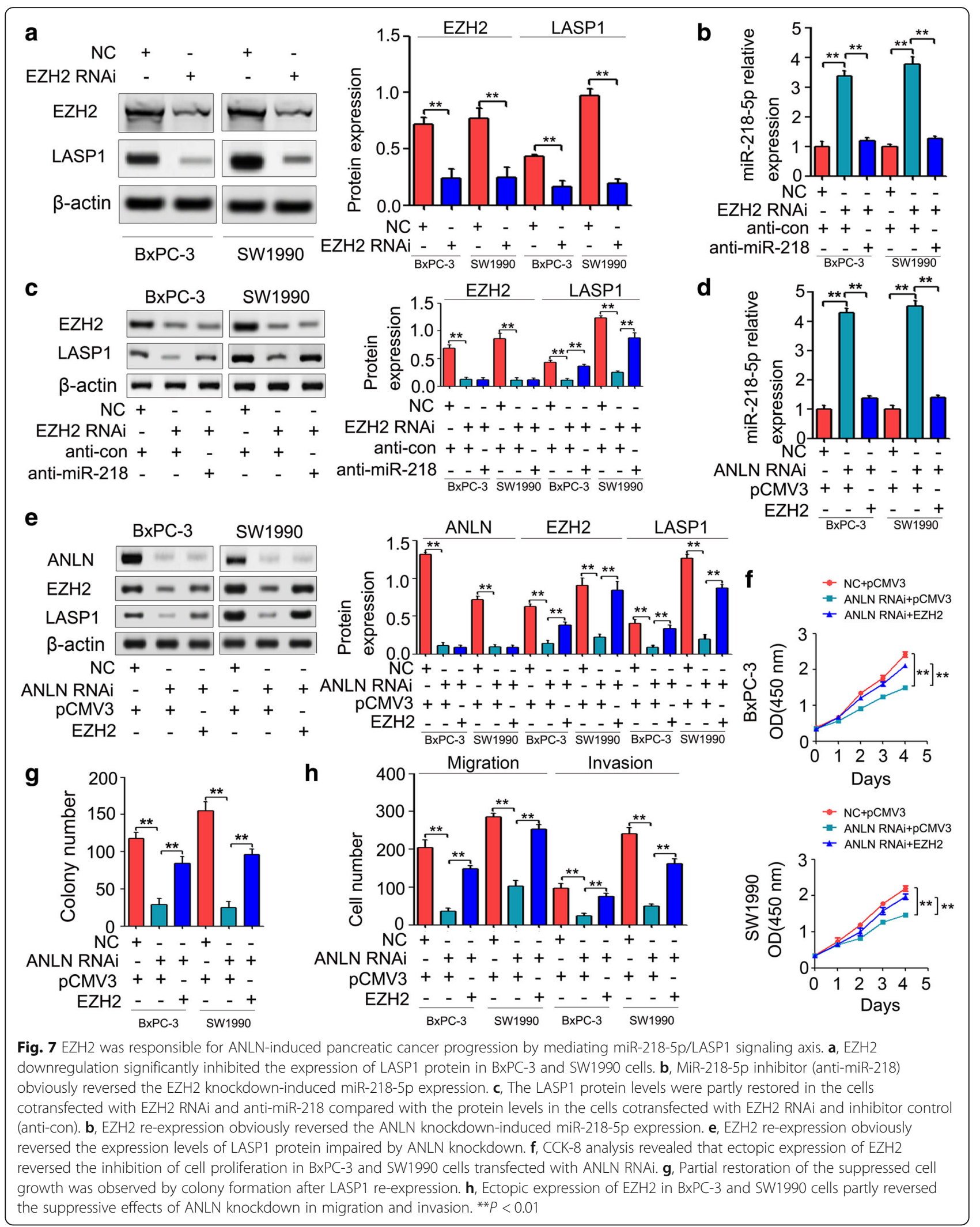




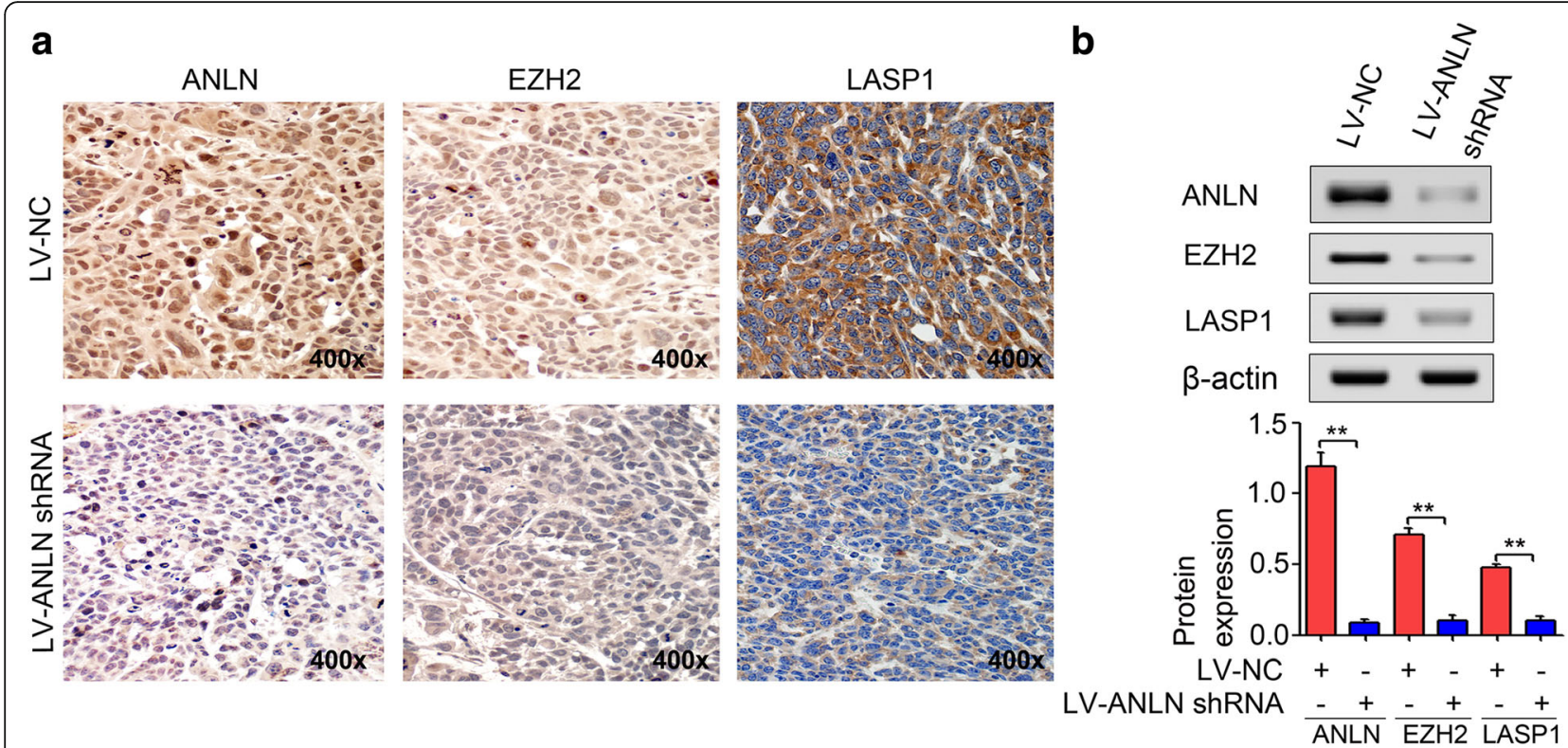

Fig. 8 ANLN knockdown repressed the levels of EZH2 and LASP1 expression in vivo. a, The levels of ANLN, EZH2 and LASP1 expression in tissues from xenograft tumor models established with the stable ANLN-silenced BxPC-3 cells or the stable scramble control BxPC-3 cells were determined by IHC. $\mathbf{b}$, Western blot analysis in tumors as in (a). ${ }^{*} P<0.01$
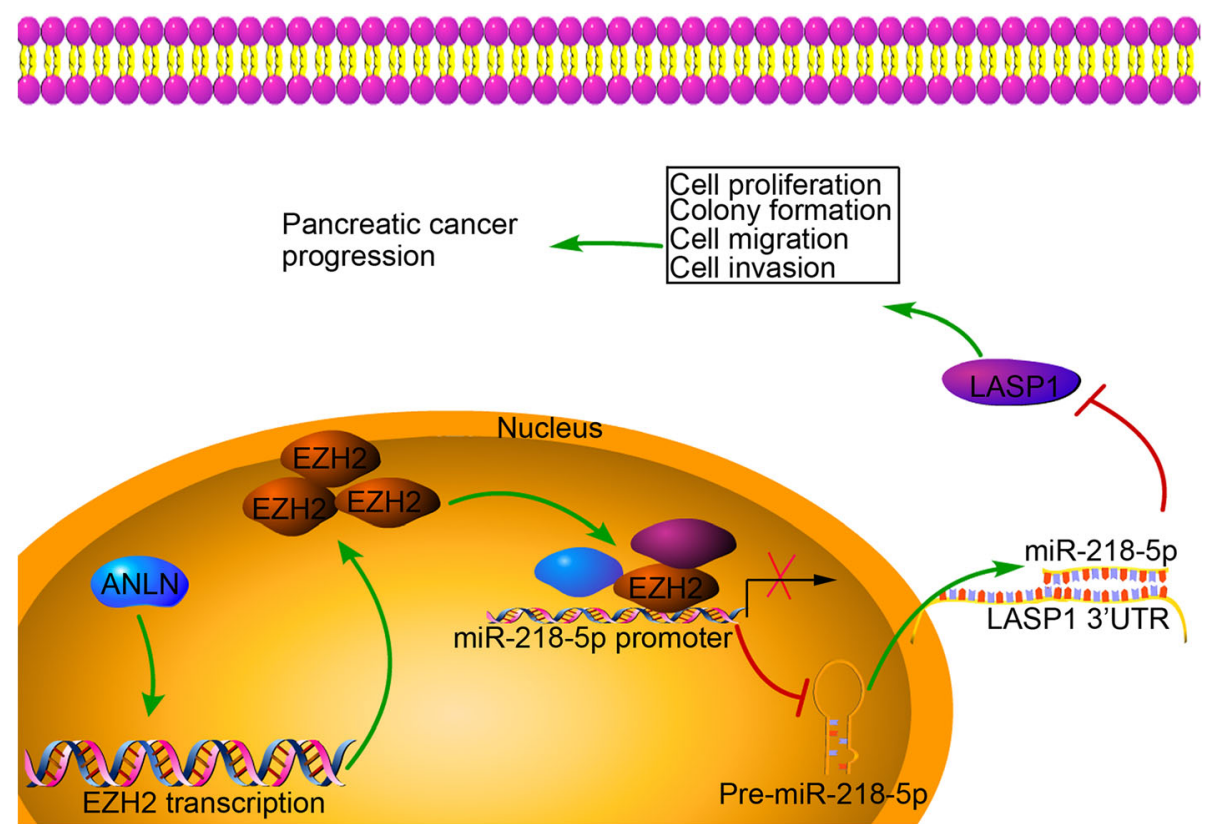

Fig. 9 Schematic diagram for the effect of ANLN on pancreatic cancer progression. ANLN upregulation promotes cell proliferation, colony formation, migration and invasion by inducing EZH2. ANLN-induced EZH2 expression leads to the downregulation of miR-218-5p, which inhibits LASP1 expression by directly targeting the 3'UTR of LASP1 
associated with a poorer outcome in patients with pancreatic cancer. Our results also showed that ANLN downregulation inhibited cell proliferation, colony formation, migration and invasion. In addition, for the first time, we identified EZH2 as a downstream effector of ANLN that positively regulates EZH2 expression. EZH2 upregulation induced by ANLN promoted pancreatic cancer cell progression by miR-218-5p/LASP1 signaling axis. Thus, ANLN may be a useful prognostic indicator and an important therapeutic target in the treatment of pancreatic cancer.

Previous studies have shown that ANLN is upregulated in many cancers and may serve as a promising prognostic biomarker for cancer [13-18]. For example, ANLN was upregulated in bladder urothelial carcinoma, and elevated ANLN expression was correlated with poor progression-free and recurrence-free survival [16]. ANLN expression was increased in pancreatic cancer, and high ANLN expression was associated with a poor prognosis in the pancreatic ductal adenocarcinoma TCGA database $[10,18]$. Consistent with previous reports, our results from the GENT database and IHC analysis showed that ANLN expression was significantly upregulated in pancreatic cancer tissues, and increased ANLN expression was significantly correlated with the tumor size, tumor differentiation, TNM stage, lymph node metastasis, distant metastasis and poorer outcome of patients with pancreatic cancer. Moreover, ANLN could serve as an independent predictor for overall survival of pancreatic cancer patients. In addition, we found that ANLN exerted tumorigenicity based on our functional studies. Consistent with our results, ANLN knockdown significantly repressed cell proliferation, migration and invasion in bladder urothelial carcinoma [16]. ANLN knockdown significantly inhibited the proliferation and migration and invasion of pancreatic cancer cells [13]. Thus, these results showed that therapeutic interventions based on ANLN regulatory strategies may be an effective way to prevent pancreatic cancer progression.

Gene microarray analysis in BxPC-3 cells transfected with ANLN siRNA showed that the genes with altered expression were highly enriched in the cell-cell adhesion and cell cycle-related biological processes (Additional file 2: Table S2, Fig. 3). It is well known that the molecules involved in cell-cell adhesion orchestrate large-scale tumor behaviors such as proliferation and invasion [42, 43]. For instance, the E3 ubiquitin ligase HUWE1 could induce cell migration and invasion by promoting cell-cell adhesion disassembly [48]. CD44 is largely involved in intracellular signaling for cell growth, proliferation and motility by mediating cellular adhesion [49]. Thus, cell-cell adhesion-related genes with altered expression in BxPC-3 cells after ANLN knockdown may play an important role in ANLN-induced pancreatic cancer cell progression. Based on GO and cluster analysis, eleven candidate genes with similar expressive trends and potential functions were selected and confirmed their expression by qRT-PCR (Fig. 3e). Among the eleven genes, four genes, including LASP1, RAB11B, RUVBL1 and MYO1B, are upregulated in human malignant tumors and promote cancer progression [20, 44-46]. Based on the GENT database, we showed that both LASP1 and RUVBL1 gene expression were significantly upregulated in pancreatic cancer tissues and were positively correlated with ANLN expression (Additional file 5: Figure S1A and B). Further investigation showed that only LASP1 restoration partially reversed the effects of ANLN knockdown on pancreatic cancer cell proliferation, colony formation, cell migration and cell invasion (Additional file 5: Figure S1C and Fig. 4). These results suggested that the upregulation of LASP1 expression induced by ANLN is partly responsible for pancreatic cancer progression.

Interestingly, our gene microarray analysis showed that 46 miRNA precursors were upregulated, and 3 miRNA precursors were downregulated (Additional file 6: Figure S2A). By combining the gene expression profiles and Targetscan (http://www.targetscan.org/), three miRNAs, including miR-145-5p, miR-218-5p and miR-9-5p, were selected and were found upregulated in ANLN downregulated BxPC-3 cells and contained binding sites of the 3'UTR of LASP1 (Additional file 6: Figure S2B), and mostly acted as tumor suppressors [50-52]. However, further investigation showed that only miR-218-5p upregulation significantly repressed LASP1 mRNA expression in BxPC-3 cells (Additional file 6: Figure S2D). Moreover, miR-218 was downregulated in pancreatic cancer, and reduced miR-218 was associated with poor prognosis of pancreatic cancer patients [29]. MiR-218 upregulation inhibited the proliferation and invasion and induced apoptosis of pancreatic cancer cells [30]. In the present study, we showed that miR-218-5p upregulation repressed pancreatic cancer cell growth, migration and invasion by directly regulating LASP1 (Fig. 5). In line with our results, miR-218-5p upregulation inhibited prostate cancer cell migration and invasion by directly regulating LASP1 expression [32]. Moreover, miR-218$5 \mathrm{p}$ downregulation partially reversed the inhibition of LASP1 expression, cell proliferation, colony formation, cell migration and cell invasion caused by ANLN knockdown (Fig. 6). These results suggested that ANLN induces the expression of LASP1 by repressing the expression of miR-218-5p, resulting in pancreatic cancer cell progression.

Additionally, EZH2-mediated H3K27 trimethylation is involved in epigenetic silencing of miR-218 [37, 53]. For example, EZH2 induces histone methylation and heterochromatin formation at miR-218-2 promoter that leads to miR-218 downregulation in pancreatic cancer, thereby mediating cell proliferation, cell migration and cell 
invasion in vitro and tumor growth and metastasis in vivo [37]. Interestingly, our results showed that ANLN knockdown significantly inhibited the expression of EZH2 in (Additional file 7: Figure S3). Moreover, miR-218-5p downregulation partially reversed the inhibition of LASP1 expression induced by EZH2 knockdown (Fig. 7c). Thus, we hypothesized that ANLN may induce the silencing of miR218-5p by mediating EZH2 in pancreatic cancer progression. Our results support this, as we found that EZH2 restoration obviously reversed the upregulation of miR-218-5p and the inhibition of LASP1 expression, cell proliferation, colony formation, cell migration and cell invasion caused by ANLN knockdown (Fig. 7d-h). We further demonstrated that ANLN knockdown significantly inhibited the levels of EZH2 and LASP1 expression in xenograft tumor models (Fig. 8). Taken together, EZH2 induced by ANLN may promote pancreatic cancer progression by regulating miR-218-5p/LASP1 signaling axis (Fig. 9).

Although previous reports and our results suggest that LASP1 contributes to pancreatic cancer cell growth and metastasis, the downstream mechanisms of LASP1 remains unclear in pancreatic cancer. Zhou $\mathrm{R}$ et al. reported that COPS5 and LASP1 synergistically interacted to induce colorectal cancer progression by PI3K/AKT pathway [20]. In non-small cell lung cancer (NSCLC), LASP1 directly bound to FAK and enhanced the phosphorylation of FAK and AKT, thereby inducing cell proliferation and invasion [54]. Gao QZ et al. found that LASP1 regulated nasopharyngeal carcinoma cell aggressiveness via LASP1/PTEN/PI3K/AKT axis [55]. In addition, PI3K/ $\mathrm{AKT}$ axis is frequently activated in pancreatic cancer and is essential for pancreatic cancer progression [56]. Therefore, vigorous research efforts are needed to further clarify whether PI3K/AKT axis contributed to LASP1-mediated pancreatic cancer cell growth and metastasis. Interestingly, previous study reported that PI3K/AKT signaling promoted the malignant potential of lung cancer cells by regulating ANLN nuclear localization and stability [17]. Thus, the role and mechanisms of PI3K/AKT signaling in regulating ANLN remained to be further elucidated.

\section{Conclusions}

In summary, we have identified the ANLN/EZH2/miR218-5p/LASP1 signaling axis in pancreatic cancer cells, which provides new insights into the mechanism underlying pancreatic cancer progression and generates a mechanism-based novel framework for developing therapeutics in pancreatic cancer treatment.

\section{Additional files}

Additional file 1: Table S1. siRNAs, shRNA and primers. (DOCX $20 \mathrm{~kb}$ )
Additional file 2: Table S2. GO terms representing biological process. (DOCX $17 \mathrm{~kb}$ )

Additional file 3: Table S3. GO terms representing molecular function. (DOCX $17 \mathrm{~kb}$ )

Additional file 4: Table S4. GO terms representing cellular compartment. (DOCX $19 \mathrm{~kb}$ )

Additional file 5: Figure S1. ANLN knockdown inhibited BXPC-3 cell proliferation by mediating LASP1. (A) Based on the GENT database, LASP1, RAB11B, RUVBL1 and MYO1B gene expression were analyzed in the pancreatic cancer tissues and normal pancreatic tissues. (B) There were significant Pearson correlations of ANLN with LASP1 and ANLN with RUVBL1 in the pancreatic cancer tissues and the normal pancreatic tissues. (C) CCK-8 assay showed that LASP1 restoration partially reversed the effects of ANLN knockdown on pancreatic cancer cell proliferation, while RUVBL1 restoration did not reverse the effect of ANLN downregulation on pancreatic cancer cell proliferation. ${ }^{* *} P<0.01$. (JPG $1806 \mathrm{~kb}$ )

Additional file 6: Figure S2. MiR-218-5p upregulation significantly repressed the expression of LASP1 mRNA in BXPC-3. (A) The heat map of the differentially expressed miRNA precursors with a fold change of greater than 2 or less than -2 in ANLN RNAi relative to NC. (B) The selected candidate miRNAs (miR-145-5p, miR-218-5p and miR-9-5p) were confirmed by qRT-PCR after ANLN RNAi transfection in BxPC-3 cells. (C) The expression levels of miR-145-5p, miR-218-5p and miR-9-5p were detected by qRT-PCR in BXPC-3 cells transfected with the miR-145-5p mimic (miR-145-5p), miR218-5p mimic (miR-218-5p), miR-9-5p mimic (miR-9-5p) or mimic control (con). U6 was used as a loading control. (D) The expression levels of LASP1 mRNA in BxPC-3 cells transfected with the miR-145-5p mimic (miR-145-5p), miR-218-5p mimic (miR-218-5p), miR-9-5p mimic (miR-9-5p) or mimic control (con) were analyzed by qRT-PCR. ${ }^{*} P<0.01$. (JPG $1590 \mathrm{~kb}$ )

Additional file 7: Figure S3. ANLN depletion significantly repressed the expression of EZH2 mRNA and protein in BxPC-3 and SW1990 cells. (A) The heat map of the selected genes. ( $\mathrm{B}$ and $\mathrm{C}$ ) The expression of EZH2 mRNA and protein was determined by qRT-PCR and Western blot in BXPC-3 and SW1990 cells transfected with the ANLN siRNA (ANLN RNAi) or the scramble control (NC). (JPG $512 \mathrm{~kb}$ )

\section{Abbreviations}

ANLN: Anillin actin binding protein; CCK-8: Cell counting kit-8; EZH2: Enhancer of zeste homolog 2; GO: Gene ontology;

IHC: Immunohistochemistry; LASP1: LIM and SH3 protein 1; MOI: Multiplicity of infection; OS: Overall survival; qRT-PCR: Quantitative reverse transcription polymerase chain reaction; shRNA: Short hairpin RNA; siRNA: Small interfering RNA; UTR: Untranslated region

\section{Acknowledgements}

Not applicable.

\section{Authors contributions}

ABW, HSD, WL and PB designed the experiments; ABW and HSD performed the experiments and analyzed the data; YG, CCZ, JJS, YDL and YJ collected and analyzed the clinical samples; ABW and HSD drafted the manuscript; WL and PB reviewed the manuscript. All authors have read and approved the final manuscript.

\section{Funding}

This study was supported by the National Natural Science Foundation of China (no. 81472288 and no. 81472775).

\section{Availability of data and materials}

All data generated or analyzed during this study are included in this published article and its additional files.

Ethics approval and consent to participate

This study was approved by the Ethical Committee of Army Medical University, China. All patients provided written informed consent to participate in the study. 


\section{Consent for publication}

The authors declare that they agree to submit the article for publication.

\section{Competing interests}

The authors declare that they have no competing interests.

Received: 3 April 2019 Accepted: 24 July 2019

Published online: 08 August 2019

\section{References}

1. Lin QJ, Yang F, Jin C, Fu DL. Current status and progress of pancreatic cancer in China. World J Gastroenterol. 2015;21(26):7988-8003.

2. Li XD, Gao PJ, Wang Y, Wang XC. Blood-derived microRNAs for pancreatic cancer diagnosis: a narrative review and meta-analysis. Front Physiol. 2018;9: 685.

3. Rossi ML, Rehman AA, Gondi CS. Therapeutic options for the management of pancreatic cancer. World J Gastroenterol. 2014;20(32):11142-59.

4. Adamska A, Falasca M. ATP-binding cassette transporters in progression and clinical outcome of pancreatic cancer: what is the way forward? World J Gastroenterol. 2018:24(29):3222-38.

5. Field CM, Alberts BM. Anillin, a contractile ring protein that cycles from the nucleus to the cell cortex. J Cell Biol. 1995:131(1):165-78.

6. Hickson GR, O'Farrell PH. Anillin: a pivotal organizer of the cytokinetic machinery. Biochem Soc Trans. 2008;36:439-41.

7. Piekny AJ, Maddox AS. The myriad roles of Anillin during cytokinesis. Semin Cell Dev Biol. 2010;21(9):881-91.

8. Oegema K, Savoian MS, Mitchison TJ, Field CM. Functional analysis of a human homologue of the Drosophila actin binding protein anillin suggests a role in cytokinesis. J Cell Biol. 2000;150(3):539-52.

9. Long XY, Zhou W, Wang YX, Liu SQ. Prognostic significance of ANLN in lung adenocarcinoma. Oncol Lett. 2018;16(2):1835-40.

10. Olakowski M, Tyszkiewicz T, Jarzab M, Król R, Oczko-Wojciechowska M, Kowalska M, Kowal M, Gala GM, Kajor M, Lange D, et al. NBL1 and anillin (ANLN) genes over-expression in pancreatic carcinoma. Folia Histochem Cytobiol. 2009:47(2):249-55.

11. Sadi AM, Wang DY, Youngson BJ, Miller N, Boerner S, Done SJ, Leong WL. Clinical relevance of DNA microarray analyses using archival formalin-fixed paraffin-embedded breast cancer specimens. BMC Cancer. 2011;11:1-13.

12. Tamura K, Furihata M, Tsunoda T, Ashida S, Takata R, Obara W, Yoshioka H, Daigo $Y$, Nasu Y, Kumon H, et al. Molecular features of hormone-refractory prostate cancer cells by genome-wide gene expression profiles. Cancer Res. 2007;67(11):5117-25

13. Wang SM, Mo YX, Midorikawa K, Zhang Z, Huang GW, Ma N, Zhao WL, Hiraku Y, Oikawa S, Murata M. The potent tumor suppressor miR-497 inhibits cancer phenotypes in nasopharyngeal carcinoma by targeting ANLN and HSPA4L. Oncotarget. 2015;6(34):35893-907.

14. Weinberger P, Ponny SR, Xu HY, Bai S, Smallridge R, Copland J, Sharma A. Cell cycle $\mathrm{M}$-phase genes are highly upregulated in anaplastic thyroid carcinoma. Thyroid. 2017;27(2):236-52.

15. Xia LL, Su XL, Shen JZ, Meng Q, Yan JQ, Zhang CH, Chen Y, Wang H, Xu MJ. ANLN functions as a key candidate gene in cervical cancer as determined by integrated bioinformatic analysis. Cancer Manag Res. 2018;10:663-70.

16. Zeng SX, Yu XW, Ma C, Song RX, Zhang ZS, Zi X, Chen X, Wang Y, Yu YW, Zhao $J$, et al. Transcriptome sequencing identifies ANLN as a promising prognostic biomarker in bladder urothelial carcinoma. Sci Rep. 2017;7(1):3151.

17. Suzuki C, Daigo Y, Ishikawa N, Kato T, Hayama S, Ito T, Tsuchiya E, Nakamura $Y$. ANLN plays a critical role in human lung carcinogenesis through the activation of RHOA and by involvement in the phosphoinositide 3-kinase/ AKT pathway. Cancer Res. 2005:65(24):11314-25.

18. Idichi T, Seki N, Kurahara H, Yonemori K, Osako Y, Arai T, Okato A, Kita Y, Arigami T, Mataki $Y$, et al. Regulation of actin-binding protein ANLN by antitumor miR-217 inhibits cancer cell aggressiveness in pancreatic ductal adenocarcinoma. Oncotarget. 2017:8(32):53180-93.

19. Tomasetto C, Régnier C, Moog-Lutz C, Mattei MG, Chenard MP, Lidereau R, Basset $P$, Rio MC. Identifcation of four novel human genes amplifed and overexpressed in breast carcinoma and localized to the q11-q21.3 region of chromosome 17. Genomics. 1995;28:367-76.

20. Zhou R, Shao ZY, Liu J, Zhan WQ, Gao QZ, Pan ZH, Wu L, Xu L, Ding YQ Zhao L. COPS5 and LASP1 synergistically interact to downregulate 14-3-30 expression and promote colorectal cancer progression via activating $\mathrm{PI} 3 \mathrm{~K}$ AKT pathway. Int J Cancer. 2018;142(9):1853-64.
21. Endres M, Kneitz S, Orth MF, Perera RK, Zernecke A, Butt E. Regulation of matrix metalloproteinases (MMPs) expression and secretion in MDA-MB-231 breast cancer cells by LIM and SH3 protein 1 (LASP1). Oncotarget. 2016;7: 64244-59.

22. Zhong $\mathrm{CH}$, Chen YT, Tao B, Peng LL, Peng TM, Yang XB, Xia XG, Chen LG. $\mathrm{LIM}$ and $\mathrm{SH} 3$ protein 1 regulates cell growth and chemosensitivity of human glioblastoma via the PI3KJAKT pathway. BMC Cancer. 2018;18(1):722.

23. Zhao L, Wang H, Liu C, Liu YW, Wang XY, Wang S, Sun XG, Li JM, Deng YJ, Jiang $Y$, Ding YQ. Promotion of colorectal cancer growth and metastasis by the LIM and SH3 domain protein 1. Gut. 2010;59:1226-35.

24. Zhao TS, Ren H, Li J, Chen J, Zhang H, Xin W, Sun Y, Sun L, Yang YW, Sun JW, et al. LASP1 is a HIF1a target gene critical for metastasis of pancreatic cancer. Cancer Res. 2015;75(1):111-9.

25. Vorvis C, Koutsioumpa M, lliopoulos D. Developments in miRNA gene signaling pathways in pancreatic cancer. Future Oncol. 2016;12(9):1135-50.

26. Liu M, Yin K, Guo X, Feng H, Yuan M, Liu Y, Zhang J, Guo B, Wang C, Zhou $\mathrm{G}$, et al. Diphthamide biosynthesis 1 is a novel oncogene in colorectal Cancer cells and is regulated by MiR-218-5p. Cell Physiol Biochem. 2017; 44(2):505-14.

27. Xu YS, He Q, Lu YY, Tao FX, Zhao L, Ou RY. MicroRNA-218-5p inhibits cell growth and metastasis in cervical cancer via LYN/NF-KB signaling pathway. Cancer Cell Int. 2018;18:198.

28. Deng M, Zeng C, Lu XH, He XS, Zhang RX, Qiu QW, Zheng GP, Jia XT, Liu H, He ZM. miR-218 suppresses gastric cancer cell cycle progression through the CDK6/cyclin D1/E2F1 axis in a feedback loop. Cancer Lett. 2017;403: 175-85.

29. Li BS, Liu H, Yang WL. Reduced miRNA-218 expression in pancreatic cancer patients as a predictor of poor prognosis. Genet Mol Res. 2015;14(4):16372-8.

30. Liu Z, Xu Y, Long J, Guo K, Ge C, Du R. microRNA-218 suppresses the proliferation, invasion and promotes apoptosis of pancreatic cancer cells by targeting HMGB1. Chin J Cancer Res. 2015;27(3):247-57.

31. Wang LL, Wang L, Wang XY, Shang D, Yin SJ, Sun LL, Ji HB. MicroRNA-218 inhibits the proliferation, migration, and invasion and promotes apoptosis of gastric cancer cells by targeting LASP1. Tumour Biol. 2016;37(11):15241-52.

32. Nishikawa R, Goto Y, Sakamoto S, Chiyomaru T, Enokida H, Kojima S, Kinoshita T, Yamamoto N, Nakagawa M, Naya Y, et al. Tumor-suppressive microRNA-218 inhibits cancer cell migration and invasion via targeting of LASP1 in prostate cancer. Cancer Sci. 2014;105(7):802-11.

33. Shahabipour F, Caraglia M, Majeed M, Derosa G, Maffioli P, Sahebkar A. Naturally occurring anti-cancer agents targeting EZH2. Cancer Lett. 2017;400:325-35

34. Yamagishi M, Uchimaru K. Targeting EZH2 in cancer therapy. Curr Opin Oncol. 2017:29(5):375-81.

35. Cao Q, Mani RS, Ateeq B, Dhanasekaran SM, Asangani IA, Prensner JR, Kim JH, Brenner JC, Jing X, Cao X, et al. Coordinated regulation of polycomb group complexes through microRNAs in cancer. Cancer Cell. 2011;20:187-99.

36. Ma J, Zhang J, Weng YC, Wang JC. EZH2-mediated microRNA-139-5p regulates epithelial-mesenchymal transition and lymph node metastasis of pancreatic Cancer. Mol Cells. 2018:41(9):868-80.

37. Li CH, To KF, Tong JH, Xiao Z, Xia T, Lai PB, Chow SC, Zhu YX, Chan SL, Marquez VE, et al. Enhancer of zeste homolog 2 silences microRNA-218 in human pancreatic ductaladenocarcinoma cells by inducing formation of heterochromatin. Gastroenterology. 2013;144(5):1086-97.

38. Kang W, Tong JH, Chan AW, Lee TL, Lung RW, Leung PP, So KK, Wu K, Fan $D, Y u$ J, et al. Yes-associated protein 1 exhibits oncogenic property in gastric cancer and its nuclear accumulation associates with poor prognosis. Clin Cancer Res. 2011;17(8):2130-9.

39. Shin G, Kang TW, Yang S, Baek SJ, Jeong YS, Kim SY. GENT: gene expression database of normal and tumor tissues. Cancer Inform. 2011:10:149-57.

40. Cerami E, Gao J, Dogrusoz U, Gross BE, Sumer SO, Aksoy BA, Jacobsen A,

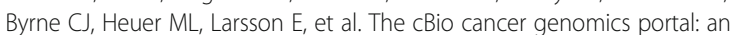
open platform for exploring multidimensional cancer genomics data. Cancer Discov. 2012;2(5):401-4

41. Gao J, Aksoy BA, Dogrusoz U, Dresdner G, Gross B, Sumer SO, Sun Y, Jacobsen $A$, Sinha R, Larsson E, et al. Integrative analysis of complex cancer genomics and clinical profiles using the cBioPortal. Sci Signal. 2013;6(269):pl1.

42. Brady-Kalnay SM. Molecular mechanisms of cancer cell-cell interactions: cellcell adhesion-dependent signaling in the tumor microenvironment. Cell Adhes Migr. 2012;6(4):344-5.

43. Hale JS, Li M, Lathia JD. The malignant social network: cell-cell adhesion and communication in cancer stem cells. Cell Adhes Migr. 2012;6(4):346-55. 
44. Taniuchi K, Furihata M, Iwasaki S, Tanaka K, Shimizu T, Saito M, Saibara T. RUVBL1 directly binds actin filaments and induces formation of cell protrusions to promote pancreatic cancer cell invasion. Int J Oncol. 2014; 44(6):1945-54

45. Lian Y, Xiong F, Yang LT, Bo H, Gong ZJ, Wang YM, Wei F, Tang YY, Li XY, Liao QJ, et al. Long noncoding RNA AFAP1-AS1 acts AS a competing endogenous RNA of miR-423-5p to facilitate nasopharyngeal carcinoma metastasis through regulating the rho/Rac pathway. J Exp Clin Cancer Res. 2018;37(1):253.

46. Zhang HR, Lai SY, Huang LJ, Zhang ZF, Liu J, Zheng SR, Ding K, Bai X, Zhou JY. Myosin 1b promotes cell proliferation, migration, and invasion in cervical cancer. Gynecol Oncol. 2018;149(1):188-97.

47. Di Leva G, Garofalo M, Croce CM. MicroRNAs in cancer. Annu Rev Pathol. 2014;9:287-314.

48. Vaughan L, Tan CT, Chapman A, Nonaka D, Mack NA, Smith D, Booton R, Hurlstone AF, Malliri A. HUWE1 ubiquitylates and degrades the RAC activator TIAM1 promoting cell-cell adhesion disassembly, migration, and invasion. Cell Rep. 2015;10(1):88-102.

49. Naor D, Nedvetzki S, Golan I, Melnik L, Faitelson Y. CD44 in cancer. Crit Rev Clin Lab Sci. 2002:39(6):527-79.

50. Ozen M, Karatas OF, Gulluoglu S, Bayrak OF, Sevli S, Guzel E, Ekici ID, Caskurlu T, Solak M, Creighton CJ, et al. Overexpression of miR-145-5p inhibits proliferation of prostate cancer cells and reduces SOX2 expression. Cancer Investig. 2015;33(6):251-8.

51. Wang J, Wang B, Ren HQ, Chen W. miR-9-5p inhibits pancreatic cancer cell proliferation, invasion and glutamine metabolism by targeting GOT1. Biochem Biophys Res Commun. 2019;509(1):241-8.

52. Fang M, Du HC, Han B, Xia GY, Shi XL, Zhang F, Fu QQ, Zhang T. Hypoxiainducible microRNA-218 inhibits trophoblast invasion by targeting LASP1: implications for preeclampsia development. Int J Biochem Cell Biol. 2017;87: 95-103.

53. Wang BR, Liu Y, Luo F, Xu Y, Qin Y, Lu XL, Xu WC, Shi L, Liu QZ, Xiang QY. Epigenetic silencing of microRNA-218 via EZH2-mediated H3K27 trimethylation is involved in malignant transformation of HBE cells induced by cigarette smoke extract. Arch Toxicol. 2016;90(2):449-61.

54. Zhang XP, Liu Y, Fan CF, Wang L, Li AL, Zhou HJ, Cai L, Miao Y, Li QC, Qiu $\mathrm{XS}$, et al. Lasp1 promotes malignant phenotype of non-small-cell lung cancer via inducing phosphorylation of FAK-AKT pathway. Oncotarget. 2017; 8(43):75102-13.

55. Gao QZ, Tang LH, Wu L, Li KT, Wang H, Li WD, Wu J, Li MY, Wang S, Zhao L. LASP1 promotes nasopharyngeal carcinoma progression through negatively regulation of the tumor suppressor PTEN. Cell Death Dis. 2018;9(3):393.

56. Ebrahimi S, Hosseini M, Shahidsales S, Maftouh M, Ferns GA, GhayourMobarhan M, Hassanian SM, Avan A. Targeting the Akt/PI3K signaling pathway as a potential therapeutic strategy for the treatment of pancreatic Cancer. Curr Med Chem. 2017;24(13):1321-31.

\section{Publisher's Note}

Springer Nature remains neutral with regard to jurisdictional claims in published maps and institutional affiliations.

Ready to submit your research? Choose BMC and benefit from:

- fast, convenient online submission

- thorough peer review by experienced researchers in your field

- rapid publication on acceptance

- support for research data, including large and complex data types

- gold Open Access which fosters wider collaboration and increased citations

- maximum visibility for your research: over $100 \mathrm{M}$ website views per year

At BMC, research is always in progress.

Learn more biomedcentral.com/submissions 